\title{
La notion de préjudice financier en dépenses
}

Mots-clés:

comptabilité publique - responsabilité personnelle et pécuniaire des comptables préjudice financier - dépenses - cour des comptes - conseil d'État

Dans deux arrêts de section du 6 décembre 2019, le Conseil d'État, juge de cassation, a précisé les grandes lignes de la notion de préjudice financier dans le cadre du contrôle de la régularité des dépenses publiques qui incombe aux comptables publics.

Nous publions ci-dessous, les deux arrêts du Conseil d'État, les conclusions du rapporteur public $M$. Dutheillet de Lamothe, que nous remercions bien vivement, et une note du professeur Xavier Vandendriessche.

\section{Conseil d'État, section, 6 décembre 2019, Mme A..., n 418741}

Vu la procédure suivante :

Par un arrêt n S2017-3987 du 5 janvier 2018, la Cour des comptes a, notamment, constitué Mme B... A..., agent comptable de l'Office national d'indemnisation des accidents médicaux, des affections iatrogènes et des infections nosocomiales (ONIAM) jusqu'au 31 janvier 2013, débitrice au titre de la charge $n^{\circ} 1$ des sommes de 20 281,82 euros et 200 euros, au titre de la charge $n^{\circ} 5$ de deux sommes de 276 euros, au titre de la charge $n^{\circ} 6$ de la somme de 276 euros, au titre de la charge $n^{\circ} 9$ de la somme de 44 012,80 euros, au titre de la charge $n^{\circ} 11$ des sommes de 9 154,14 euros et 80138,05 euros, au titre de la charge $n^{\circ} 17$ des sommes de 136 857,44 euros, 217 810,84 euros et 44 326,48 euros augmentées des intérêts de droit.

Considérant ce qui suit :

1. Aux termes du I de l'article 60 de la loi du 23 février 1963 de finances pour 1963, dans sa rédaction issue de l'article 90 de la loi du 28 décembre 2011 de finances rectificative pour 2011, qui définit les obligations qu'il incombe au comptable public de respecter sous peine de voir sa responsabilité personnelle et pécuniaire engagée : "Outre la responsabilité attachée à leur qualité d'agent public, les comptables publics sont personnellement et pécuniairement responsables du recouvrement des recettes, du paiement des dépenses, de la garde et de la conservation des fonds et valeurs appartenant ou 
confiés aux différentes personnes morales de droit public dotées d'un comptable public, désignées ci-après par le terme d'organismes publics, du maniement des fonds et des mouvements de comptes de disponibilités, de la conservation des pièces justificatives des opérations et documents de comptabilité ainsi que de la tenue de la comptabilité du poste comptable qu'ils dirigent. / Les comptables publics sont personnellement et pécuniairement responsables des contrôles qu'ils sont tenus d'assurer en matière de recettes, de dépenses et de patrimoine dans les conditions prévues par le règlement général sur la comptabilité publique. / La responsabilité personnelle et pécuniaire prévue ci-dessus se trouve engagée dès lors qu'un déficit ou un manquant en monnaie ou en valeurs a été constaté, qu'une recette n'a pas été recouvrée, qu'une dépense a été irrégulièrement payée ou que, par le fait du comptable public, I'organisme public a dû procéder à l'indemnisation d'un autre organisme public ou d'un tiers ou a dû rétribuer un commis d'office pour produire les comptes. / (...) «. Aux termes du VI du même article : «La responsabilité personnelle et pécuniaire prévue au l est mise en jeu par le ministre dont relève le comptable, le ministre chargé du budget ou le juge des comptes dans les conditions qui suivent. (...) / Lorsque le manquement du comptable aux obligations mentionnées au I n'a pas causé de préjudice financier à l'organisme public concerné, le juge des comptes peut l'obliger à s'acquitter d'une somme arrêtée, pour chaque exercice, en tenant compte des circonstances de l'espèce. Le montant maximal de cette somme est fixé par décret en Conseil d'État en fonction du niveau des garanties mentionnées au II. / Lorsque le manquement du comptable aux obligations mentionnées au I a causé un préjudice financier à l'organisme public concerné ou que, par le fait du comptable public, l'organisme public a dû procéder à l'indemnisation d'un autre organisme public ou d'un tiers ou a dû rétribuer un commis d'office pour produire les comptes, le comptable a l'obligation de verser immédiatement de ses deniers personnels la somme correspondante. / (...) «. Aux termes du IX du même article : « Les comptables publics dont la responsabilité personnelle et pécuniaire a été mise en jeu dans les cas mentionnés au deuxième alinéa du VI ne peuvent obtenir du ministre chargé du budget la remise gracieuse des sommes mises à leur charge. / Les comptables publics dont la responsabilité personnelle et pécuniaire a été mise en jeu dans les cas mentionnés au troisième alinéa du même VI peuvent obtenir du ministre chargé du budget la remise gracieuse des sommes mises à leur charge. Hormis le cas de décès du comptable ou de respect par celui-ci, sous l'appréciation du juge des comptes, des règles de contrôle sélectif des dépenses, aucune remise gracieuse totale ne peut être accordée au comptable public dont la responsabilité personnelle et pécuniaire a été mise en jeu par le juge des comptes, le ministre chargé du budget étant dans l'obligation de laisser à la charge du comptable une somme au moins égale au double de la somme mentionnée au deuxième alinéa dudit VI. / (...) «. Aux termes de l'article 12 du décret du 29 décembre 1962 portant règlement général sur la comptabilité publique, alors applicable : « Les comptables sont tenus d'exercer : / (...) B. - En matière de dépenses, le contrôle : / De la qualité de l'ordonnateur ou de son délégué ; / De la disponibilité des crédits; / De l'exacte imputation des dépenses aux chapitres qu'elles concernent selon leur nature ou leur objet ; / De la validité de la créance dans les conditions prévues à l'article 13 ci-après ; / Du caractère libératoire du règlement. (...) «. Enfin, selon l'article 13 du même décret, alors applicable : «En ce qui concerne la validité de la créance, le contrôle porte sur: / La justification du service fait et l'exactitude des calculs de liquidation; / L'intervention préalable des contrôles réglementaires et la production des justifications. / En outre, dans la mesure où les règles propres à chaque organisme public le prévoient, les comptables publics vérifient l'existence du visa des contrôleurs financiers sur les engagements et les ordonnancements émis par les ordonnateurs principaux. / Les comptables publics vérifient également l'application des règles de prescription et de déchéance. «. Aux termes de l'article 19 du décret du 7 novembre 2012 relatif à la gestion budgétaire et comptable publique, dans sa rédaction alors applicable : « Le comptable public est tenu d'exercer le contrôle : (...) $2^{\circ}$ S'agissant des ordres de payer : / a) De la qualité de l'ordonnateur ; / b) De l'exacte imputation des dépenses au regard des règles relatives à la spécialité des crédits ; / c) De la disponibilité des crédits ; / d) De la validité de la dette dans les conditions prévues à l'article 20 ; / e) Du caractère libératoire du paiement ; (...). «. Enfin, selon l'article 20 du même décret, dans sa rédaction alors applicable : « Le contrôle des comptables publics sur la validité de la dette porte sur : / $1^{\circ}$ La justification du service fait ; / $2^{\circ} \mathrm{L}^{\prime}$ exactitude de la liquidation ; / $3^{\circ} \mathrm{L}^{\prime}$ intervention des contrôles préalables prescrits par la réglementation; $/ 4^{\circ}$ Dans la mesure où les règles propres à chaque personne morale mentionnée à l'article

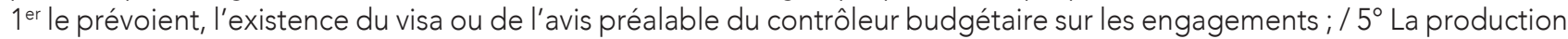

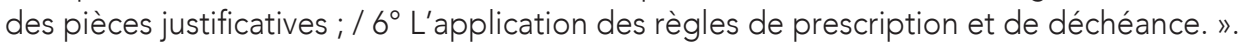

2. Les dispositions rappelées ci-dessus instituent, dans l'intérêt de l'ordre public financier, un régime légal de responsabilité pécuniaire et personnelle des comptables publics distinct de la responsabilité de droit commun. Lorsque le manquement du comptable aux obligations qui lui incombent n'a pas causé de préjudice financier à l'organisme public concerné, le juge des comptes peut l'obliger à s'acquitter d'une somme non rémissible. Lorsque le manquement du comptable a causé un préjudice financier à l'organisme public concerné, le juge des comptes met en débet le comptable qui a alors l'obligation de verser de ses deniers personnels la somme correspondante. Il appartient ainsi au juge des comptes d'apprécier si le manquement du comptable a causé un préjudice financier à l'organisme public concerné et, le cas échéant, d'évaluer l'ampleur de ce préjudice. II doit, à cette fin, d'une part, rechercher s'il existait un lien de causalité entre le préjudice et le manquement à la date où ce dernier a été commis, et, d'autre part, apprécier le montant du préjudice à la date à laquelle il statue en prenant en compte, le cas échéant, des éléments postérieurs au manquement.

3. Pour déterminer si le paiement irrégulier d'une dépense par un comptable public a causé un préjudice financier à l'organisme public concerné, il appartient au juge des comptes de vérifier, au vu des éléments qui lui sont soumis à la date 
à laquelle il statue, si la correcte exécution, par le comptable, des contrôles lui incombant aurait permis d'éviter que soit payée une dépense qui n'était pas effectivement due. Lorsque le manquement du comptable porte sur l'exactitude de la liquidation de la dépense et qu'il en est résulté un trop-payé, ou conduit à payer une dépense en l'absence de tout ordre de payer ou une dette prescrite ou non échue, ou à priver le paiement d'effet libératoire, il doit être regardé comme ayant par lui-même, sauf circonstances particulières, causé un préjudice financier à l'organisme public concerné. A l'inverse, lorsque le manquement du comptable aux obligations qui lui incombent au titre du paiement d'une dépense porte seulement sur le respect de règles formelles que sont l'exacte imputation budgétaire de la dépense ou l'existence du visa du contrôleur budgétaire lorsque celle-ci devait, en l'état des textes applicables, être contrôlée par le comptable, il doit être regardé comme n'ayant pas par lui-même, sauf circonstances particulières, causé de préjudice financier à l'organisme public concerné. Le manquement du comptable aux autres obligations lui incombant, telles que le contrôle de la qualité de l'ordonnateur ou de son délégué, de la disponibilité des crédits, de la production des pièces justificatives requises ou de la certification du service fait, doit être regardé comme n'ayant, en principe, pas causé un préjudice financier à l'organisme public concerné lorsqu'il ressort des pièces du dossier, y compris d'éléments postérieurs aux manquements en cause, que la dépense repose sur les fondements juridiques dont il appartenait au comptable de vérifier l'existence au regard de la nomenclature, que l'ordonnateur a voulu l'exposer, et, le cas échéant, que le service a été fait.

4. En premier lieu, au titre de la charge $n^{\circ} 9$, la Cour des comptes a constitué Mme A..., agent comptable de l'Office national d'indemnisation des accidents médicaux, des affections iatrogènes et des infections nosocomiales (ONIAM), débitrice pour la somme de 44 012,80 euros augmentée des intérêts de droit, au titre de l'exercice 2012, pour avoir procédé au règlement de factures correspondant à des prestations réalisées par une société informatique alors qu'elle ne disposait pas des éléments requis en vertu de la réglementation et du contrat signé avec cette société permettant de vérifier l'exactitude des calculs de liquidation de la dette. Toutefois, il résulte de ce qui a été dit aux points 2 et 3 qu'il appartient au juge des comptes, pour déterminer si le paiement irrégulier d'une dépense a causé un préjudice financier à l'organisme public concerné, d'apprécier si ces dépenses étaient effectivement dues en tenant compte, le cas échéant, des éléments justificatifs produits postérieurement au manquement. Par suite, en se fondant, pour caractériser l'existence d'un préjudice financier pour l'établissement résultant du caractère irrégulier de ces paiements, sur le caractère insuffisant des seuls éléments dont disposait la comptable publique à la date du manquement, sans rechercher s'il ressortait des pièces du dossier qui lui était soumis, et notamment des pièces produites devant elle en défense par la comptable, que les paiements litigieux pouvaient être regardés comme effectivement dus, la Cour a entaché son arrêt d'erreur de droit.

5. En second lieu, il ressort des pièces du dossier soumis aux juges du fond que Mme A... a pris en charge, sur le fondement d'une convention conclue par l'Office avec une société de voyages, des mandats portant sur un montant total de 398 994,76 euros au titre des exercices 2011, 2012 et 2013 correspondant à des prestations fournies par cette société à l'Office. Au titre de la charge $n^{\circ} 17$, la Cour des comptes a relevé, par l'arrêt attaqué, que la comptable avait produit, d'une part, une convention d'ouverture de compte « voyages d'affaires « entre l'Office et la société, datée de 2003 et comportant le visa du contrôleur général économique et financier mais ne prévoyant pas de prix pour les prestations susceptibles d'être fournies, et, d'autre part, un document en date du 4 mars 2005 fixant la rémunération applicable aux différentes prestations offertes par cette société, cosigné par le secrétaire général de l'Office et valant accord des parties, document dont elle a retenu qu'il ne constituait pas une pièce justificative valide de la dépense faute de visa du contrôleur général économique et financier. Elle a alors jugé que, en l'absence de ce visa sur ce document, les prix des différentes prestations étaient non justifiés et les paiements effectués en contrepartie des prestations indus. II résulte toutefois de ce qui a été dit au point 3 qu'en se bornant à ces constatations pour caractériser l'existence d'un préjudice financier résultant de ces paiements irréguliers alors que le seul défaut de vérification du visa du contrôleur budgétaire par le comptable n'est pas, en lui-même, de nature à causer un préjudice financier à l'organisme public concerné, la Cour a entaché son arrêt d'erreur de droit.

6. II résulte de tout ce qui précède, sans qu'il soit besoin d'examiner l'autre moyen du pourvoi, que Mme A... est fondée à demander l'annulation de l'arrêt attaqué en tant que, par ses articles 12 et 23, il l'a constituée débitrice, respectivement, d'une somme de 44 012,80 euros augmentée des intérêts de droit au titre de l'exercice 2012, et d'une somme totale de 398 994,76 euros augmentée des intérêts de droit au titre des exercices 2011, 2012 et 2013.

\section{DECIDE:}

Article 1er: Les articles 12 et 23 de l'arrêt de la Cour des comptes du 5 janvier 2018 sont annulés.

Article 2 : L'affaire est renvoyée, dans cette mesure, à la Cour des comptes.

Article 3 : La présente décision sera notifiée à Mme B... A... et au parquet général près la Cour des comptes.

Copie en sera adressée au ministre de l'action et des comptes publics et à l'Office national d'indemnisation des accidents médicaux, des affections iatrogènes et des infections nosocomiales. 


\section{Conseil d'État, section, 6 décembre 2019, ministre de l'action et des comptes publics, $n^{\circ} 425542$}

Vu la procédure suivante :

Par un arrêt n S 2018-2690 du 5 octobre 2018, la Cour des comptes a, notamment, constitué M. B... A..., directeur régional des finances publiques de Bretagne et du département d'llle-et-Vilaine, débiteur du Trésor, au titre de la charge $n^{\circ}$ 6, de la somme de 113 275,33 euros, augmentée des intérêts de droit à compter du 27 novembre 2017. Par un pourvoi et un mémoire en réplique, enregistrés les 20 novembre 2018 et 1 er août 2019 au secrétariat du contentieux du Conseil d'État, le ministre de l'action et des comptes publics demande au Conseil d'État:

$\cdots$

Considérant ce qui suit :

1. Aux termes du I de l'article 60 de la loi du 23 février 1963 de finances pour 1963, dans sa rédaction issue de l'article 90 de la loi du 28 décembre 2011 de finances rectificative pour 2011, qui définit les obligations qu'il incombe au comptable public de respecter sous peine de voir sa responsabilité personnelle et pécuniaire engagée : «Outre la responsabilité attachée à leur qualité d'agent public, les comptables publics sont personnellement et pécuniairement responsables du recouvrement des recettes, du paiement des dépenses, de la garde et de la conservation des fonds et valeurs appartenant ou confiés aux différentes personnes morales de droit public dotées d'un comptable public, désignées ci-après par le terme d'organismes publics, du maniement des fonds et des mouvements de comptes de disponibilités, de la conservation des pièces justificatives des opérations et documents de comptabilité ainsi que de la tenue de la comptabilité du poste comptable qu'ils dirigent. / Les comptables publics sont personnellement et pécuniairement responsables des contrôles qu'ils sont tenus d'assurer en matière de recettes, de dépenses et de patrimoine dans les conditions prévues par le règlement général sur la comptabilité publique. / La responsabilité personnelle et pécuniaire prévue ci-dessus se trouve engagée dès lors qu'un déficit ou un manquant en monnaie ou en valeurs a été constaté, qu'une recette n'a pas été recouvrée, qu'une dépense a été irrégulièrement payée ou que, par le fait du comptable public, l'organisme public a dû procéder à l'indemnisation d'un autre organisme public ou d'un tiers ou a dû rétribuer un commis d'office pour produire les comptes. / (...) «. Aux termes du VI du même article : "La responsabilité personnelle et pécuniaire prévue au I est mise en jeu par le ministre dont relève le comptable, le ministre chargé du budget ou le juge des comptes dans les conditions qui suivent. (...) / Lorsque le manquement du comptable aux obligations mentionnées au I n'a pas causé de préjudice financier à l'organisme public concerné, le juge des comptes peut l'obliger à s'acquitter d'une somme arrêtée, pour chaque exercice, en tenant compte des circonstances de l'espèce. Le montant maximal de cette somme est fixé par décret en Conseil d'État en fonction du niveau des garanties mentionnées au II. / Lorsque le manquement du comptable aux obligations mentionnées au I a causé un préjudice financier à l'organisme public concerné ou que, par le fait du comptable public, l'organisme public a dû procéder à l'indemnisation d'un autre organisme public ou d'un tiers ou a dû rétribuer un commis d'office pour produire les comptes, le comptable a l'obligation de verser immédiatement de ses deniers personnels la somme correspondante. / (...) «. Aux termes du IX du même article : « Les comptables publics dont la responsabilité personnelle et pécuniaire a été mise en jeu dans les cas mentionnés au deuxième alinéa du VI ne peuvent obtenir du ministre chargé du budget la remise gracieuse des sommes mises à leur charge. / Les comptables publics dont la responsabilité personnelle et pécuniaire a été mise en jeu dans les cas mentionnés au troisième alinéa du même VI peuvent obtenir du ministre chargé du budget la remise gracieuse des sommes mises à leur charge. Hormis le cas de décès du comptable ou de respect par celui-ci, sous l'appréciation du juge des comptes, des règles de contrôle sélectif des dépenses, aucune remise gracieuse totale ne peut être accordée au comptable public dont la responsabilité personnelle et pécuniaire a été mise en jeu par le juge des comptes, le ministre chargé du budget étant dans l'obligation de laisser à la charge du comptable une somme au moins égale au double de la somme mentionnée au deuxième alinéa dudit VI. / (...) «. Aux termes de l'article 12 du décret du 29 décembre 1962 portant règlement général sur la comptabilité publique, alors applicable : «Les comptables sont tenus d'exercer : / (...) B. - En matière de dépenses, le contrôle : / De la qualité de l'ordonnateur ou de son délégué ; / De la disponibilité des crédits ; / De l'exacte imputation des dépenses aux chapitres qu'elles concernent selon leur nature ou leur objet; / De la validité de la créance dans les conditions prévues à l'article 13 ci-après ; / Du caractère libératoire du règlement. (...) «. Enfin, selon l'article 13 du même décret, alors applicable : «En ce qui concerne la validité de la créance, le contrôle porte sur: / La justification du service fait et l'exactitude des calculs de liquidation ; / L'intervention préalable des contrôles réglementaires et la production des justifications. / En outre, dans la mesure où les règles propres à chaque organisme public le prévoient, les comptables publics vérifient l'existence du visa des contrôleurs financiers sur les engagements et les ordonnancements émis par les ordonnateurs principaux. / Les comptables publics vérifient également l'application des règles de prescription et de déchéance. 
2. Les dispositions rappelées ci-dessus instituent, dans l'intérêt de l'ordre public financier, un régime légal de responsabilité pécuniaire et personnelle des comptables publics distinct de la responsabilité de droit commun. Lorsque le manquement du comptable aux obligations qui lui incombent n'a pas causé de préjudice financier à l'organisme public concerné, le juge des comptes peut l'obliger à s'acquitter d'une somme non rémissible. Lorsque le manquement du comptable a causé un préjudice financier à l'organisme public concerné, le juge des comptes met en débet le comptable qui a alors l'obligation de verser de ses deniers personnels la somme correspondante. II appartient ainsi au juge des comptes d'apprécier si le manquement du comptable a causé un préjudice financier à l'organisme public concerné et, le cas échéant, d'évaluer l'ampleur de ce préjudice. II doit, à cette fin, d'une part, rechercher s'il existait un lien de causalité entre le préjudice et le manquement à la date où ce dernier a été commis, et, d'autre part, apprécier le montant du préjudice à la date à laquelle il statue en prenant en compte, le cas échéant, des éléments postérieurs au manquement.

3. Pour déterminer si le paiement irrégulier d'une dépense par un comptable public a causé un préjudice financier à l'organisme public concerné, il appartient au juge des comptes de vérifier, au vu des éléments qui lui sont soumis à la date à laquelle il statue, si la correcte exécution, par le comptable, des contrôles lui incombant aurait permis d'éviter que soit payée une dépense qui n'était pas effectivement due. Lorsque le manquement du comptable porte sur l'exactitude de la liquidation de la dépense et qu'il en est résulté un trop-payé, ou conduit à payer une dépense en l'absence de tout ordre de payer ou une dette prescrite ou non échue, ou à priver le paiement d'effet libératoire, il doit être regardé comme ayant par lui-même, sauf circonstances particulières, causé un préjudice financier à l'organisme public concerné. A l'inverse, lorsque le manquement du comptable aux obligations qui lui incombent au titre du paiement d'une dépense porte seulement sur le respect de règles formelles que sont l'exacte imputation budgétaire de la dépense ou l'existence du visa du contrôleur budgétaire lorsque celle-ci devait, en l'état des textes applicables, être contrôlée par le comptable, il doit être regardé comme n'ayant pas par lui-même, sauf circonstances particulières, causé de préjudice financier à l'organisme public concerné. Le manquement du comptable aux autres obligations lui incombant, telles que le contrôle de la qualité de l'ordonnateur ou de son délégué, de la disponibilité des crédits, de la production des pièces justificatives requises ou de la certification du service fait, doit être regardé comme n'ayant, en principe, pas causé un préjudice financier à l'organisme public concerné lorsqu'il ressort des pièces du dossier, y compris d'éléments postérieurs aux manquements en cause, que la dépense repose sur les fondements juridiques dont il appartenait au comptable de vérifier l'existence au regard de la nomenclature, que l'ordonnateur a voulu l'exposer et, le cas échéant, que le service a été fait.

4. Il ressort des pièces du dossier soumis aux juges du fond que M. B... A..., directeur régional des finances publiques de Bretagne et du département d'llle-et-Vilaine, a pris en charge trois mandats pour un montant total de 113 275,33 euros au titre de l'exercice 2011, sur le fondement d'un contrat de marché public et de bons de commande signés par des personnes habilitées à engager ces dépenses pour le compte de l'organisme public concerné, et correspondant à des prestations exécutées. Au titre de la charge $n^{\circ} 6$, la Cour des comptes a relevé que ces paiements étaient intervenus alors que leur montant était supérieur à la délégation consentie aux délégués de l'ordonnateur. Elle a alors jugé que le paiement d'un mandat signé par un ordonnateur non habilité constituait une dépense indue, même en présence de service fait, que ne figurait au dossier aucune indication attestant de la volonté de l'ordonnateur compétent de consentir une délégation aux signataires à hauteur des montants réglés et que, ainsi, le manquement du comptable avait causé un préjudice au Trésor. II résulte toutefois de ce qui a été dit au point 3 qu'en se fondant sur l'absence de volonté de l'ordonnateur d'accorder une délégation de signature aux signataires des ordres de payer pour caractériser l'existence d'un préjudice financier résultant des paiements en litige alors, d'une part, qu'il est constant que ces paiements correspondaient à des prestations exécutées sur la base d'un contrat de marché public et de bons de commande et, d'autre part, qu'était établie, par la production du contrat de marché public et des bons de commande, la volonté de l'ordonnateur d'exposer ces dépenses, la Cour a entaché son arrêt d'erreur de droit.

5. Il résulte de ce qui précède, sans qu'il soit besoin d'examiner l'autre moyen du pourvoi, que le ministre de l'action et des comptes publics est fondé à demander l'annulation de l'arrêt attaqué en tant que, par son article 10, il a constitué M. A... débiteur de la somme de 113 275,33 euros augmentée des intérêts de droit au titre de l'exercice 2011.

\section{DECIDE:}

Article $1^{\text {er }}$ : L'article 10 de l'arrêt de la Cour des comptes du 5 octobre 2018 est annulé.

Article 2 : L'affaire est renvoyée, pour cette mesure, à la Cour des comptes.

Article 3 : La présente décision sera notifiée au ministre de l'action et des comptes publics et au parquet général près la Cour des comptes.

Copie en sera adressée et à M. B... A.... 


\section{CONCLUSIONS}

\section{Louis DUTHEILLET de LAMOTHE, rapporteur public Conseil d'État, section, 6 décembre 2019,n¹8741 et 425542}

1. En vertu de l'article 60 de la loi du 23 février 1963 de finances pour 1963, les comptables publics sont personnellement et pécuniairement responsables du recouvrement des recettes, du paiement des dépenses et de la conservation des fonds et valeurs des personnes morales de droit public pour lesquelles ils officient. Cette responsabilité de plein droit, si lourde dans son principe, les conduit à devoir rembourser sur leurs deniers personnels la recette non recouvrée, la dépense irrégulière et tout manquant dans la caisse. Cependant, depuis l'intervention de l'article 90 de la loi du 28 décembre 2011 de finances rectificative pour 2011, ils ne sont désormais tenus au paiement de ce débet que lorsque le manquement commis a causé un préjudice financier à l'organisme public. Dans le cas inverse, une somme dite irrémissible, qui nous semble avoir une vocation répressive, est mise à leur charge « en tenant compte des circonstances de l'espèce ». Cette distinction est fondamentale puisque la somme irrémissible est souvent limitée à quelques centaines d'euros, tandis qu'il y a des exemples de débets en dizaines de millions d'euros (C.Comptes, 8 mars 2018, n²018-0513).

Ces deux affaires ont été inscrites à votre formation de jugement afin que soit précisé dans quelles hypothèses les manquements comptables dans le paiement des dépenses de l'administration doivent être regardés comme causant un préjudice financier aux collectivités publiques. Vous avez apporté une réponse claire et simple s'agissant des manquements des comptables dans le recouvrement des recettes, en jugeant par votre décision de section du 27 juillet 2015 (CE, sect., 27 juillet 2015, Min chargé du budget c/ parquet général de la Cour des comptes, $n^{\circ}$ 370430, Rec.) qu'un tel manquement « doit en principe être regardé comme ayant causé un préjudice financier à l'organisme public concerné », sauf si à la date du manquement la dette " était irrécouvrable en raison notamment de l'insolvabilité de la personne qui en était redevable». Sans que votre jurisprudence ait eu à le préciser jusqu'ici, il nous semble également clair qu'en cas de manquant dans la caisse du comptable, le préjudice financier est établi de ce fait même. Reste le cas des dépenses irrégulières.
Nous aimerions pouvoir vous proposer aujourd'hui une réponse aussi nette, mais c'est impossible du fait de la variété des dépenses et de la diversité des contrôles attendus des comptables sur ces opérations. Face à une telle question, aux multiples ramifications, deux démarches jurisprudentielles sont possibles. Une approche inductive qui par petites touches, réglant chaque cas d'espèce, dessine prudemment et progressivement les modalités d'application de la règle. C'est l'approche traditionnelle et elle est souvent opportune. C'est celle qui a été suivie jusqu'ici par la Cour des comptes et par vous-mêmes. Pour la question qui nous occupe aujourd'hui, cette méthode ne s'est pas avérée satisfaisante: la réforme aura bientôt huit ans et la question continue d'agiter la doctrine, alors qu'elle est d'une importance capitale pour les comptables et pour l'exercice par la Cour des comptes de son office de juge des comptes. Dans un article paru en avril 2017, M. le président Girardi, magistrat financier, admettait ainsi « qu'il est pour l'instant difficile de préciser quels critères devraient être appliqués à chaque cas particulier » et que «face à des décisions contradictoires, les praticiens mais surtout les justiciables demeurent dans l'incertitude sur les arguments à faire valoir pour influencer l'appréciation du juge des comptes » (J-L-Girardi, Les incertitudes nées de l'application de la réforme du régime de responsabilité personnelle et pécuniaire des comptables publics, GFP, n²-2017, p. 89). ${ }^{1}$

Ce constat nous semble encore valable et c'est pourquoi nous allons vous proposer, à partir de deux affaires instruites par votre sixième chambre, d'adopter aujourd'hui une démarche consistant à décrire dans vos décisions à venir ces " critères généraux » appelés de ses vœux par la doctrine, et qui dans l'idéal devraient permettre de déduire, pour tous les manquements et toutes les dépenses, s'il y a ou non préjudice financier. Pour tenter d'y parvenir, nous allons procéder en trois temps : il faut d'abord revenir sur l'objectif de la réforme et les difficultés qu'elle pose ; nous vous présenterons ensuite votre jurisprudence actuelle; et nous tenterons enfin de cerner les critères du préjudice financier en matière de dé- 
pense en fonction des différentes sortes de manquements comptables.

2. Tout est pourtant parti d'une idée très simple, une idée qui paraissait commandée tant par le bon sens que par le souci de protéger les droits fondamentaux des comptables. La responsabilité personnelle et pécuniaire des comptables est en effet l'héritage d'un système aussi efficace que rigoureux de protection des deniers publics, dont les racines remontent au bas-moyen âge. II consiste à diviser les rôles entre trois acteurs, en distinguant celui qui décide de la dépense mais ne peut la réaliser, qui est devenu l'ordonnateur ; le comptable qui ne peut la décider mais a seul accès à la caisse pour payer la dépense; et le juge des comptes qui procède à la reddition des comptes et, s'il constate la moindre irrégularité, supprime la somme en question des écritures comptables, de la ligne de compte, et met à la charge du comptable le paiement d'une somme dite débet, qui permet de restaurer la caisse publique au niveau théorique où le compte ainsi corrigé par le juge des écritures irrégulières prévoit qu'elle devrait être. Le débet constitue une puissante incitation à la rigueur des contrôles.

La sévérité de ce dispositif de responsabilité de plein droit, qui fait de tout manquement du comptable dans son service une faute personnelle, sauf en cas de force majeure, est depuis longtemps assumée par le législateur, qui l'a maintenu jusqu'à nos jours. Mais la pratique et la doctrine avaient démontré le caractère excessif des règles en vigueur avant 2011 dans I'hypothèse de ce qu'il était convenu d'appeler les débets sans préjudice. Si, en effet, une commune acquiert un véhicule et que le comptable commet des irrégularités tenant à une erreur d'imputation de la somme au bon chapitre de sa comptabilité, à l'absence d'une pièce justificative requise ou encore au paiement sans justificatif $d u$ service fait, c'est-à-dire de ce que la voiture est bien acquise à la collectivité, le comptable pouvait avant 2011 se retrouver en débet du montant de la voiture. Et pourtant, la voiture que souhaitait acquérir la commune est dans la cour de la mairie et elle est utilisée par les agents. Comme le comptable ne récupérait pas la voiture dont il remboursait le prix à la commune..., la commune bénéficiait d'une voiture gratuite : il y avait manifestement enrichissement sans cause. D'aucuns émettaient même des doutes sur la proportionnalité, et donc la conventionnalité ou constitutionnalité du dispositif, quand il aboutissait à mettre à la charge des comptables des sommes colossales correspondant à des immeubles ou travaux pourtant acquis par les collectivités pu- bliques. Ce qui était censée être une forme de réparation du compte se transformait de facto en une peine, prononcée sans considérations du comportement du comptable et des circonstances ni prise en compte de ses facultés contributives. En pratique, le ministre était d'ailleurs conduit à accorder presque systématiquement des remises gracieuses pour les débets importants.

Les travaux préparatoires de la loi du 28 décembre 2011 témoignent clairement de ce que la réforme vise à remédier à ce problème, par une formule simple qui limite le prononcé de débets aux cas où le manquement du comptable a causé un préjudice financier à l'organisme public, et prévoit dans les autres cas le paiement d'une somme d'argent indépendante du montant des opérations irrégulières. Cette révolution fondamentale dans les conditions d'engagement de la responsabilité des comptables, qui transforme encore un peu plus l'office du juge des comptes en un juge des comptes et des comptables, constitue un progrès important. Ayant ainsi recentré les débets sur les cas où la responsabilité du comptable répond à la réparation d'un préjudice, le législateur a aussi souhaité encadrer le pouvoir de remise gracieuse du ministre, en rendant irrémissible la somme payée en l'absence de préjudice et en limitant les possibilités de remise gracieuse en cas de débets. En revanche, ces travaux ne donnent pas d'indication claire sur les critères du préjudice financier, sinon que cette notion repose sur l'intuition qu'il est injuste de faire payer au comptable une somme correspondant à une contrepartie dont continue de jouir la personne publique.

Cette idée simple doit constituer le fil d'Ariane dans la définition du préjudice financier. Cependant, lorsqu'on rentre dans le détail des cas d'espèces, selon les manquements et selon les dépenses, on se rend compte que cette notion de préjudice financier est difficile à cerner et qu'on hésite souvent. Pour l'illustrer, il suffit de présenter les cas d'espèces des affaires examinées aujourd'hui. Le premier pourvoi $n^{\circ} 418741$, pour lequel restent en litige les charges $n^{\circ} 9$ et 17 , est relatif à la gestion comptable de I'ONIAM par Mme A.... La charge 9 est relative au paiement de factures de prestations informatiques, sans les justificatifs requis, à savoir le contrat pour le premier paiement et l'équivalent d'un bon de commande pour les suivants, et alors que la facture n'était pas régulière faute de comporter les détails suffisants pour vérifier les calculs de liquidation. La cour des comptes a estimé « qu'en l'absence des éléments de liquidation de la dette et même de 
la période d'exécution des prestations », contrairement à ce qu'exige la réglementation sur les factures, la facture n'aurait pas dû être réglée par le comptable et qu'il y a donc eu préjudice financier. A l'inverse, la comptable se pourvoit en cassation car elle estime avoir prouvé, en produisant au juge des comptes le contrat et des éléments établissant la réalité des prestations fournies, que le service avait été fait et correspondait au montant réglé, entraînant l'absence de préjudice.

Au titre de la charge $n^{\circ} 17$, il est reproché au même comptable d'avoir réglé une série de mandats correspondant aux prestations d'une société organisant des voyages d'affaire, pour un montant total de près de 400000 euros. La cour a estimé que le comptable avait irrégulièrement payé ces dépenses car la lettre cosignée par les parties et fixant le barème des prix n'avait pas été visée par le contrôleur budgétaire, ce dont le comptable aurait dû s'assurer. La cour a jugé que « en l'absence de [ce] visa, cette convention qui fonde les conditions de paiement d'une partie de ces prestations, ne constitue pas une pièce justificative de la dépense ». Selon la cour, une dépense dont les prix n'étaient pas régulièrement établis ne pouvait pas être exposée et cause donc un préjudice financier, quand bien même les prestations ont été fournies. Le comptable soutient bien sûr que la production de documents établissant l'accord des parties et la réalité du service fait devaient suffire à établir l'absence de préjudice.

Enfin, dans le second pourvoi $n^{\circ} 425542$, le comptable, directeur régional des finances publiques de Bretagne et d'Ile-et-Vilaine, a été mis en débet pour avoir exécuté trois mandats d'un montant dépassant celui pour lequel le signataire avait reçu délégation de l'ordonnateur, pour un total de 113275 euros. ${ }^{2}$ Le manquement est incontestable, mais le service fait l'est tout autant et le comptable estime qu'il n'y a pas eu préjudice. La cour a jugé que « le paiement d'un mandat signé par un ordonnateur non habilité constitue par principe une dépense indue » et que, dès lors que rien n'attestait de la volonté de l'ordonnateur de déléguer à ces agents une délégation à hauteur de tels montants, le trésor avait subi un préjudice. II s'agit d'une position de principe qu'elle a déjà prise dans des précédents. ${ }^{3}$

Relevons que pour la plupart des autres charges, qui ne sont plus en litige, la cour a écarté l'existence d'un préjudice financier, en estimant notamment qu'une dépense payée sans production du contrat était irrégulière mais $n$ 'avait pas en l'espèce causé de préjudice financier au motif que l'acte avait été produit par la suite, était régulier au regard des règles comptables et qu'ainsi « le fondement juridique de la dépense est établi ».

3. Cette notion de "fondement juridique » est au centre du débat, car elle constitue la boussole qu'a jusqu'ici fourni votre jurisprudence, mais son maniement s'est avéré malaisé. II nous faut donc maintenant vous présenter l'état de la jurisprudence, qu'il s'agit aujourd'hui de préciser et non de remettre en cause.

Votre décision de section du 27 juillet 2015, qui a traité plus spécifiquement des opérations de recettes, a tout d'abord précisé qu'il convenait de se placer à la date du manquement pour apprécier s'il causait un préjudice, mais qu'il était possible, et ce point est essentiel, d'établir l'existence ou l'inexistence du préjudice par des documents postérieurs au manquement.

Une riche décision de chambres réunies du 26 mai 2016 de la Cour des comptes, qui sert souvent de référence au parquet financier et à la doctrine 4 a fixé des repères, les charges examinées permettant d'aborder un grand nombre $d$ 'hypothèses (Cour des comptes, chambres réunies, 26 mai 2016, Direction régionale des finances publiques de Rhône-Alpes, nº S-20161602). La lecture de la décision de la Cour des comptes montre que celle-ci, sans s'essayer à une théorie générale, s'appuie alternativement sur trois éléments pour conclure à l'existence ou non d'un préjudice : la réalité du service fait, le caractère dû ou indu de la dépense et la volonté de l'ordonnateur.

C'est ensuite une décision de chambres réunies du Conseil d'État du 22 février 2017, relative à la comptabilité du Grand port maritime de Rouen, qui a posé les jalons du raisonnement à tenir en cas d'irrégularité comptable dans le paiement d'une dépense. Elle procède en deux temps. Vous avez d'abord insisté sur le fait que le juge des comptes doit s'interroger successivement sur l'existence d'un manquement et sur l'existence d'un lien de causalité entre ce manquement et un préjudice financier, à la date du manquement. S'agissant ensuite du paiement irrégulier d'une dépense, vous avez jugé que, pour apprécier l'existence du préjudice, « il appartient au juge des comptes d'apprécier si la dépense était effectivement due et, à ce titre, de vérifier notamment qu'elle n'était pas dépourvue de fondement juridique ». En l'espèce, il s'agissait du cas d'une dépense payée alors que le marché public était arrivé à son terme. Vous avez censuré la cour pour avoir déduit un préjudice de cette seule circonstance, en relevant qu'une intention manifeste des parties de poursuivre leurs 
relations contractuelles pouvait révéler l'existence d'un contrat oral, ce que vous admettez (CE, section, 8 février 2012, Min. du budget, $n^{\circ}$ 340698, Rec. ; CE, 4 mai 2015, Sté Bueil publicité, $n^{\circ} 371455$, inédit), ou que des avenants rétroactifs pouvaient, en matière contractuels, reconstituer le fondement juridique manquant. Vous avez par ailleurs précisé dans cette décision qu'il appartenait au juge des comptes, s'il fixe un débet, d'actualiser pour ce faire le montant du préjudice au jour où il statue.

Enfin, pour terminer ce tableau, signalons qu'une décision antérieure, du 27 mai 2015 (CE, Ministre délégué, chargé du budget $c /$ Parquet général près la Cour des comptes, $n^{\circ} 374708$, T.) avait jugé, s'agissant du cas particulier des primes, pour lequel votre jurisprudence témoigne d'une attention particulière, que le paiement d'une indemnité non instituée par un texte législatif ou réglementaire, dont la référence doit d'après les nomenclatures être fournie au comptable avec l'ordre de payer, cause en principe un préjudice financier « eu égard au paiement indu de ce paiement».

4. Sur le plan des principes et du vocabulaire, on constate une grande convergence entre votre jurisprudence et celle de la cour : la dépense irrégulière causant un préjudice financier est celle qui n'est "pas effectivement due », et un élément central de cette appréciation est l'existence d'un «fondement juridique», lequel est apprécié assez souplement en matière de contrat, plus rigoureusement s'agissant d'un fondement réglementaire. Mais l'application de ces notions aux cas d'espèce fait trop souvent débat. Peut-on aujourd'hui, s'essayer à fixer des critères plus précis? Non sans quelques craintes de mal embrasser une réalité aussi diverse, nous allons vous le proposer, en procédant en deux étapes. La première consiste à repartir des éléments constitutifs du débet dans le nouveau régime de responsabilité ; la seconde consistera à les appliquer à chaque forme de manquement comptable en matière de dépense, en testant ces critères sur divers exemples de dépenses.

4.1 II est certain que pour prononcer un débet, il faut désormais trois éléments : un manquement comptable, pour lequel la jurisprudence est ancienne, un " préjudice financier », notion difficile à caractériser, et un lien de causalité. II nous semble que le lien de causalité est un élément cardinal de cette nouvelle législation et qu'il a parfois été perdu de vue : les huit années de pratique ont montré qu'il n'est pas possible d'apprécier le préjudice financier pour lui-même, de façon absolue, comme le préjudice de droit commun. II s'agit d'une notion sui generis. Alors qu'en droit de la responsabilité de droit commun on peut apprécier faute, préjudice et causalité de façon relativement distincte, en statuant parfois sur un seul de ces éléments, il nous semble qu'ici il ne faut pas se demander, dans l'absolu, si la collectivité publique souffre financièrement : la recherche du préjudice soit partir d'une interrogation sur les conséquences qu'a eu le manquement du comptable par lui-même. II faut remettre le lien de causalité au centre de l'analyse, au lieu d'essayer d'autonomiser la notion de préjudice financier en la réduisant à une dépense qui n'est pas effectivement due. II faut réunir les deux éléments séparés dans la rédaction de l'arrêt Grand port maritime de Rouen pour les saisir ensemble.

Si on ne peut pas partir de la seule notion de dépense non effectivement due pour apprécier le préjudice financier, c'est qu'il est au contraire, parfois, du devoir du comptable de payer une dépense qui n'est pas due : vous affirmez depuis toujours que le comptable ne doit pas s'interroger de façon générale sur la légalité d'une dépense mais se borner aux contrôles limitatifs qui constituent la régularité comptable de l'opération et circonscrivent l'ordre public financier qu'il doit protéger (CE, Assemblée, 12 juillet 1907, Ministre des finances c/ Nicolle, $n^{\circ} 23933$, p. 670 ; CE, 5 février 1971, Ministre de l'économie et des finances c/ M. Balme, $n^{\circ} 71173$, p. 105). Ces contrôles peuvent le conduire à s'interroger sur certaines questions de légalité, par exemple lorsque les nomenclatures comptables lui enjoignent de bloquer le paiement de primes s'il $n^{\prime}$ est pas fait mention du " texte institutif de l'indemnité ", mais au-delà des contrôles comptables, la dépense décidée par l'ordonnateur doit être payée, même si elle n'est pas due, même si elle est illégale. L'ordonnateur engage sa responsabilité propre, et le comptable n'a pas pour mission de se faire le censeur de ses choix.

L'appréciation d'un lien de causalité entre un manquement comptable et un préjudice financier nous semble donc devoir s'apprécier à I'aune de la question hypothétique suivante: si le comptable n'avait pas manqué à ses contrôles, $s^{\prime} i l$ avait correctement rempli son office en exigeant les pièces et les informations nécessaires, la dépense aurait-elle quand même pu être exposée ? Si c'est le cas, son manquement n'a pas causé préjudice aux finances de l'organisme, quand bien même on s'apercevrait ensuite que la dépense est illégale, qu'elle n'est pas « effectivement due ". II y a eu une irrégularité comptable, et elle a peut-être eu des conséquences importantes : peut-être la dépense n'aurait-elle 
jamais eu lieu en présence d'un comptable plus rigoureux. Le comptable sera donc condamné à payer une somme irrémissible. Mais parce qu'elle pouvait avoir lieu et qu'elle a eu lieu, même irrégulière, avec sa contrepartie, le comptable n'a pas à la rembourser intégralement. Si à l'inverse, le contrôle comptable devait dans un tel cas empêcher que la dépense irrégulière soit exposée, pour des vices suffisamment substantiels et non régularisables, il doit la rembourser. ${ }^{5}$ Dans cette reconstitution, vous avez accepté que le juge tienne compte d'éléments postérieurs pour établir, notamment, si le service a été fait, quels sont les fondements juridiques de la dépense, et si I'ordonnateur voulait bien l'exposer.

4.2 Puisque la démarche que nous vous proposons part du manquement, et non du préjudice, pour apprécier ce qui peut être regardé comme causé par le manquement, et le distinguer de ce qui est causé par les choix, même illégaux, de I'ordonnateur, nous vous proposons maintenant de passer en revue les différents manquements possibles. II suffit pour cela de partir de la liste de base des contrôles comptables pour les opérations de dépense, fixées aux articles 19 et 20 du décret du 7 novembre 2012 relatif à la gestion budgétaire et comptable publique, qui trouvaient leur équivalent dans l'ancien décret du 29 décembre 1962 (les deux textes étant applicables en l'espèce, selon les charges), cette liste étant adaptée par des dispositions particulières. Pour certains des contrôles, et donc certains des manquements, il nous semble qu'on peut affirmer de façon générale qu'en principe, sauf circonstance exceptionnelle, ils causent ou ne causent pas un préjudice financier. C'est ce que le professeur Damarey a désigné comme l'apparition de «théorèmes financiers » dans la jurisprudence comptable (S. Damarey, Le préjudice financier, nouveau critère de la responsabilité du comptable public, AJDA 2017, p. 1435 ; S. Damarey, Le préjudice financier devant le juge des comptes, Droit administratif, n 8-9, août 2018, 5 - Lexisnexis).

Les plus évidents de ces "théorèmes " correspondent aux cas où le manquement crée structurellement un préjudice. Nous identifions quatre manquements de cette sorte : l'erreur sur la liquidation faite à partir des éléments régulièrement soumis au comptable, qui conduit à un troppayé. Il en va de même du paiement d'une dette prescrite ou non échue, ou encore d'un paiement qui n'a pas d'effet libératoire, notamment lorsqu'il n'est pas adressé au véritable créancier de l'administration, ce dont le comptable doit s'assurer. Enfin, il nous semble qu'une dépense réglée en l'absence de tout ordre de payer doit être regardée comme causant en principe un préjudice, hypothèse qu'il faut distinguer de celle de l'espèce, où il y a un ordre de payer mais dont le signataire avait excédé sa délégation. Si véritablement il n'a pas d'ordre de payer, même pas oral (ce qui serait irrégulier mais attesterait de la volonté de l'ordonnateur), cela signifie par définition que l'argent ne devait pas sortir de la caisse publique et que le comptable doit le rembourser. Nous avons hésité à vous proposer d'ajouter à cette liste le cas de l'indisponibilité des crédits au chapitre budgétaire correspondant à la dépense. II nous semble cependant plus difficile de tenir une position de principe, dès lors que l'article 7 de loi organique sur les lois de finances $n^{\circ} 2001$ 692 du 1er août 2001 a consacré une importante fongibilité des crédits entre les titres.

Il est moins facile de définir des cas où le manquement doit en principe être regardé comme ne causant pas de préjudice. La doctrine a plusieurs fois souligné qu'une erreur dans l'imputation de la dépense au bon chapitre budgétaire devrait être regardée comme ne causant pas, en principe, de préjudice financier. ${ }^{6}$ Néanmoins, une erreur d'imputation pourra dans certains causer un préjudice, notamment si la correcte imputation aurait conduit à estimer les crédits radicalement indisponibles. La jurisprudence devra aussi se prononcer sur l'hypothèse du préjudice indirect, qui n'est pas celui correspondant à la dépense exposée, mais qui est dû aux conséquences de second ordre, notamment s'agissant du calcul de taxes ou de subventions et dotations pour l'organisme public.

Le second des pourvois pose la question délicate du défaut de visa ou d'avis du contrôleur budgétaire. Ce point de contrôle du comptable, qui était autrefois systématique, n'est plus prévu que pour certaines catégories d'organismes depuis I'intervention du décret $n^{\circ} 2018-803$ du 24 septembre 2018, dès lors que l'intervention de cette forme de tutelle budgétaire a vocation à contrôler la soutenabilité de l'exécution du budget et non la légalité ou la régularité comptable des opérations. ${ }^{7}$ La doctrine a plutôt pris parti en faveur du caractère formel de ce contrôle, dont la mauvaise exécution doit être regardée comme ne causant pas de préjudice financier (cf. Girardi et Renouard, 2012, prec.) mais la jurisprudence de la Cour des comptes semble en sens inverse lorsque le visa ou l'avis requis fait effectivement défaut. Cette position pourrait se justifier par le fait que l'intervention du contrôleur budgétaire aurait pu conduire l'ordonnateur à renoncer à une dépense inconsidérée et que, faute de certitude que la dépense aurait été exposée, un pré- 
judice doit être retenu. C'est pousser trop loin à nos yeux l'exigence de certitude que la dépense aurait été exposée : toutes choses égales par ailleurs, en présence d'une dépense dont le seul défaut est de n'avoir pas été soumise au contrôleur budgétaire, il nous semble qu'il faut considérer qu'il n'y a pas de préjudice financier. II en va de même pour l'ensemble de ce qui se rattachait à la catégorie des « contrôles préalables prescrits par la réglementation », qui ont été supprimés par le même décret du 24 septembre 2018.

4.3 Ici s'arrête, à nos yeux, la liste de ces théorèmes. Pour tous les autres cas de figure, la situation apparaît plus contrastée. La pratique de la cour des comptes a montré que l'appréciation du préjudice dépend fortement du cas d'espèce et des justificatifs produits a posteriori par le comptable. Pour ces manquements, relatifs à la qualité de l'ordonnateur, à la disponibilité des crédits, à la certification du service fait et aux pièces justificatives, il vous faut donc préciser des critères. Il nous semble qu'il est possible de dessiner un ensemble de conditions qui, si elles sont remplies, permettront d'affirmer qu'il n'y a pas de préjudice, sauf peut-être dans des circonstances exceptionnelles, à réserver par prudence.

Le premier de ces critères relève de l'évidence, c'est l'existence du service fait, c'est-à-dire de la contrepartie de la dépense. C'est parce qu'il y a une voiture dans la cour de la mairie qu'on peut estimer que le comptable, qui l'a payée irrégulièrement, n'a pas à la rembourser, mais devra seulement payer la somme irrémissible, cette amende qui ne dit pas son nom. Et la question se pose de savoir si on ne devrait pas s'arrêter là : l'idée que le comptable ne doit pas rembourser une dépense qui a eu une contrepartie, pour éviter un enrichissement sans cause de la collectivité publique, est l'âme de la réforme, et s'en tenir là aboutirait à un dispositif particulièrement simple. Nous pensons cependant que c'est impossible, comme vous l'avez d'ailleurs déjà jugé par votre décision Grand port maritime de Rouen en exigeant qu'on vérifie que la dépense n'est pas par ailleurs dépourvue de fondement juridique. Nous voyons deux obstacles à cette simplification extrême. Le premier est qu'il existe des dépenses sans service fait, sans contrepartie, telles les aides publiques de toutes sortes, les subventions ou les primes versées aux agents qui n'ont pas de contrepartie individualisable. Le second est plus fondamental : il existe, à notre avis, un niveau d'irrégularité de la dépense qui devient trop grave, trop aigu, pour qu'elle puisse être regardée comme n'ayant pas causé un préjudice aux finances publiques, quand bien même cette dépense a acheté quelque chose. C'est ce que vous avez exprimé par l'idée que la dépense qui pas de fondement juridique cause un préjudice financier.

Mais la pratique a montré que cette expression s'avérait difficile à manier, pouvant aboutir à des exigences telles que l'effet recherché de la réforme s'en trouve trop amoindri. Les cas d'espèce le montrent. Il faut préciser cette notion. Une solution consisterait à s'en tenir au fondement de premier rang, c'est-à-dire l'acte qui traduit l'engagement juridique de la dépense ordonnancée : le contrat, voire le seul bon de commande, suffirait ainsi pour une dépense contractuelle, quelle qu'elle soit. Mais cette solution simple heurterait ce que vous avez jugé, à bon droit nous semble-t-il, pour les primes: le paiement d'une prime, pourtant décidée par l'ordonnateur (ce serait là le fondement de premier rang), mais en dehors de tout texte institutif, cause par principe un préjudice. A l'extrême inverse, le juge des comptes pourrait vérifier l'existence de toute la chaine de fondements qui va du texte législatif ou réglementaire à l'ordre de payer en passant par des délibérations du conseil d'administration, des décisions de l'autorité exécutive, et peut-être une convention ou d'autre actes. Mais on tomberait alors dans un contrôle, au moins partiel, de la légalité de la dépense qui excèderait l'office du comptable. Cela ne serait pas conforme à la démarche que nous vous proposons : ne mettre à la charge du comptable que le paiement d'une somme indue qu'il aurait dû bloquer, et pas les sommes, même illégales, qu'il aurait pu régulièrement payer. II faut donc, nous semble-t-il, aligner la recherche du fondement juridique qui permet d'écarter l'existence d'un préjudice sur l'étendue des justificatifs que la nomenclature applicable imposait au comptable de réclamer. Parmi ces justificatifs, il en est qui ne servent pas à fonder la dépense, parce qu'ils ont trait au service fait, permettent de préciser l'imputation budgétaire, l'identité du créancier, ou apportent des précisions non essentielles ou procédurales. Mais la plupart d'entre eux tendent à établir la validité de la dette. Ce sont ces éléments dont le juge devra vérifier si on peut considérer, a posteriori, qu'ils sont réunis, à la mesure de ce que prévoit la nomenclature. Ainsi, lorsque la nomenclature ne prévoit que la production au comptable du contrat, il doit simplement s'interroger sur l'existence d'un contrat qui, nous l'avons vu, peut-être oral ou rétroactif mais qui permet bien d'apporter un fondement juridique à la dépense de la collectivité. Si une délibération de l'organe délibérant était légalement nécessaire pour signer ce contrat et n'existe pas, cela 
ne conduira pas à considérer qu'il y a préjudice, car le comptable aurait dû payer cette dépense sur le fondement du seul contrat, même illégal. En revanche, lorsque la nomenclature exige, pour certaines catégories de dépenses, la délibération de l'organe délibérant l'autorisant, le juge des comptes ne pourra écarter l'existence d'un préjudice que si une telle délibération lui est produite. Autre exemple: pour une subvention, la signature d'une convention avec le subventionné est obligatoire au-dessus d'un certain montant : dès lors que la nomenclature prévoit qu'elle doit être produite au comptable pour le versement de la subvention, nous pensons que le préjudice ne peut être écarté que si, au moment où le juge statue, une telle convention existe : elle précise les conditions d'emploi de la somme et les engagements du subventionné, elle fonde bien la dépense publique. L'approche par la nomenclature permet en outre d'intégrer indirectement des considérations relatives à l'importance et à la fréquence de la dépense en cause, car la nomenclature adapte l'importance des justificatifs à la sensibilité de la dépense.

Nous voudrions faire deux remarques sur cette approche des fondements juridiques de la dépense.

Ces quelques exemples montrent d'abord à quel point l'admissibilité des éléments postérieurs au manquement revêt un caractère crucial. Votre décision de section de 2015 a insisté sur ce point et votre décision Grand port maritime de Rouen a souligné, pour un contrat, qu'il fallait prendre en compte des " avenants rétroactifs » à un contrat arrivé à son terme. Le manquement s'apprécie le jour où le comptable a effectué ses contrôles et au regard des seules pièces qu'il avait à l'époque. Le lien de causalité avec un préjudice s'apprécie à la même date, mais en prenant en compte tous les éléments en possession du juge le jour où il statue. ${ }^{8}$ Ce qui permet d'écarter le préjudice, ce n'est pas la production des pièces justificatives manquantes datées du jour où le comptable a payé, c'est la justification de ce que la dépense peut être regardée comme fondée. Le comptable aurait certes dû la bloquer dans un premier temps, mais on aurait pu l'exposer ensuite sans irrégularité comptable. Cette rétroactivité connaît probablement des limites : vous auriez du mal à admettre que, pour une prime distribuée sans texte, on prenne en compte un texte réglementaire rétroactif, puisque la rétroactivité et la régularisation ne sont en principe pas admises en cette matière. Vous n'avez pas aujourd'hui à théoriser cette question, mais il faut insister sur son importance pratique dans le maniement de la notion de préjudice financier.

En second lieu, il faut souligner que ce critère du fondement juridique que vous avez dégagé en 2017 absorbe souvent le critère de la volonté, souvent évoqué dans la doctrine et repris parfois par la cour des comptes, puisque les pièces qui établissent le fondement juridique témoignent d'une volonté d'engager la dépense. Y a-t-il lieu cependant d'ajouter aux critères du service fait et des fondements juridiques un troisième critère se rattachant à la volonté d'exposer la dépense?

Nous pensons qu'il n'est pas nécessaire s'agissant de la volonté de l'organisme public dont le comptable à la charge, car cette question renvoie à la répartition des compétences à l'intérieur de la personne publique. Or vous jugez de façon constante que le comptable n'a pas à vérifier la correcte application des règles de répartition des compétences ou le respect des délégations (CE, 4 mai 2018, Min de l'action et des comptes publics, $n^{\circ} 410880$, T. ; CE, 28 décembre 2018, SDIS de la Gironde, n 410113, T.), s'agissant de l'engagement administratif de la dépense. Autrement dit, si la nomenclature exige de lui qu'il vérifie l'existence d'une délibération du conseil d'administration autorisant, par exemple la signature d'un contrat, comme c'est généralement le cas, par exemple, pour les contrats de partenariat, le préjudice ne sera écarté que si cette délibération est produite devant le juge des comptes, ce qui établira à la fois fondement et volonté. Si en revanche un tel justificatif n'est pas exigé, le comptable n'avait pas à s'intéresser à l'accord de l'organe délibérant et aurait dû exécuter le mandat de l'ordonnateur y compris en cas d'absence illégale de cette délibération. II n'y a alors pas de raison de rechercher la volonté de l'organisme au stade de l'examen du préjudice.

En revanche, il nous semble utile que vous précisiez qu'il faut toujours s'interroger sur la volonté de l'ordonnateur. Certes, la plupart du temps, comme pour la volonté de l'organisme lui-même, elle ressortira des pièces établissant que la dépense peut être regardée comme fondée juridiquement. Mais ce n'est pas toujours le cas, notamment lorsqu'il s'agit d'une dette préexistante, d'un droit qu'un usager tire d'un texte ou d'une dépense directement décidée par une délibération de l'organe délibérant. Dans de tel cas, si l'ordre de payer a été signé par une personne incompétente, qui excède notamment la délégation qui lui a été consentie, comme en l'espèce, il ne peut y avoir absence de préjudice que s'il n'y avait pas opposition de l'ordonnateur à ce qu'il soit procédé à cette dépense. En effet, rien 
ne doit conduire à passer outre à la volonté de I'ordonnateur de décider d'une dépense. Seraitelle-même indubitablement due que le comptable qui la règle cause un préjudice financier à la collectivité dont il a la charge : elle n'aurait jamais pu être réglée sans l'accord de l'ordonnateur, qui prend sa responsabilité si son refus est finalement jugé illégal. II nous semble donc utile de conserver un troisième critère du préjudice financier tenant à la volonté de l'ordonnateur, même s'il sera souvent redondant avec celui du fondement juridique.

Nous avons en revanche écarté d'autres critères qui ont pu être avancés, y compris par certains arrêts de la cour. Nous avons un moment pensé qu'il fallait distinguer entre les cas où la dépense correspond à une dette préexistante dont la personne publique s'acquitte et ceux où elle décide librement d'engager la dépense. Mais cette distinction est plus pertinente du point de vue de I'ordonnateur que du comptable, pour qui toute dépense se présente comme un ordre de payer de l'ordonnateur, quelle que soit l'origine de la dette, si vous nous suivez pour juger qu'il y a en principe préjudice lorsque le comptable a payé une somme que l'ordonnateur ne voulait pas exposer. Nous n'avons pas non plus retenu une distinction selon que la nomenclature prévoit que le comptable doit disposer ou non de l'original d'un acte ou qualifié de conforme à l'original, distinction sur laquelle s'est appuyé l'arrêt de chambres réunies de la cour des comptes de 2016 (Cour des comptes, chambres réunies, 26 mai 2016, Direction régionale des finances publiques de Rhône-Alpes, $n^{\circ}$ S-2016-1602). Votre pratique nous semble engagée pour accepter devant le juge, dans l'esprit de la réforme, la production d'éléments de nature à suppléer l'absence ou la perte de l'original qui aurait dû être produit à l'époque.

5. En conclusion, il nous semble donc que lorsque la dépense irrégulière a trouvé sa contrepartie, s'il y en a une, peut être regardée comme fondée juridiquement et comme ayant eu l'accord de l'ordonnateur, le juge des comptes doit en principe écarter l'existence d'un préjudice et ne pas prononcer de débet. Ces critères sont cohérents avec les cas où nous vous avons proposé de retenir l'existence ou l'inexistence systématique d'un préjudice. La variété des cas est telle qu'il vous faut probablement réserver des cas exceptionnels où il en irait autrement, mais ils nous semblent rares.

Au terme de cette présentation, il nous faut terminer en revenant aux arrêts déférés et en tirer des conséquences sur les pourvois. Si vous nous avez suivis, vous constaterez que, dans leur vocabulaire et sur le plan des principes, ce que nous vous proposons s'inscrit dans la continuité de ce que juge la Cour des comptes. Mais, dans l'application de ces notions, vous serez conduits à censurer des exigences excessives au titre de la justification du fondement juridique de la dépense ou de la volonté de l'ordonnateur. Ainsi :

- dans le premier pourvoi, le seul fait que la comptable n'avait pas d'élément pour procéder à une liquidation correcte ne permet pas de conclure à l'existence d'un préjudice, et en l'espèce, le contrat de prestations informatiques existait même s'il avait été perdu et que le comptable ne l'avait pas; en outre, le seul défaut de visa du contrôleur budgétaire ne crée en principe pas de préjudice financier.

- dans le second pourvoi, la cour a commis une erreur de droit en refusant que l'absence d'ordres de payer signés par un délégataire compétent ne soit palliée par tous éléments établissant la volonté de l'ordonnateur de l'époque d'exposer cette dépense, sans qu'il y ait à établir une volonté de déléguer sa signature pour les montants en cause.

Par ces motifs nous concluons donc, sous le $n^{\circ} 418741$, à l'annulation des articles 12 et 23 de l'arrêt de la Cour des comptes du 5 janvier 2018 , et sous le $n^{\circ} 425542$, à l'annulation de l'article 10 de l'arrêt du 5 octobre 2018, les deux affaires devant être renvoyées dans cette mesure à la cour. 


\title{
La responsabilité du comptable et le préjudice financier en dépenses, Note sous CE, 6 décembre 2019, ns 418741 et 425542 , concl. L. Dutheillet de Lamothe
}

\author{
Xavier VANDENDRIESSCHE, Professeur à Sciences-Po Lille
}

La réforme des conditions d'engagement de la responsabilité personnelle et pécunaire du comptable introduite par la loi n²011-1978 du 28 déc. 2011 (art. 90) continue d'agiter la doctrine et les prétoires les plus prestigieux, de celui de la Cour des comptes à celui de son juge de cassation. Le législateur a souhaité limiter le prononcé d'un débet par le juge des comptes aux hypothèses dans lesquelles le manquement du comptable a entraîné un préjudice financier pour l'organisme public en cause.

Il s'agissait de remédier à un effet pervers du système de responsabilité du comptable, qualifié de "débet sans préjudice »; fort du caractère objectif de son office, le juge des comptes considérait en effet que la responsabilité personnelle et pécuniaire du comptable public s'apprécie en fonction des obligations que lui imposent la législation et la réglementation nationale et communautaire ${ }^{9}$ et non le préjudice ou l'enrichissement sans cause qui pourrait résulter des actes dudit comptable ou des décisions des juridictions financières ${ }^{10}$. Cette situation était doublement contestable : inéquitable pour le comptable tout d'abord dès lors que la dépense payée était bien voulue par la personne publique et que la somme était bien due par elle dès lors que le service dont elle était la contrepartie avait bien été effectué ; exagérément favorable à la personne publique en cause qui pouvait ainsi bénéficier d'un enrichissement sans cause. Ainsi, le « manquant » dans la caisse du comptable n'était que juridique et non réel.

Certes, ce mauvais système comportait un correctif : le pouvoir de remise gracieuse reconnu au ministre. Toutefois, on aboutissait ainsi, pour des raisons purement pratiques, à ce que l'administration puisse priver d'effet une décision juridictionnelle rendue par une juridiction indépendante.

La réforme de 2011 était donc salutaire et attendue même si la solution retenue par la nouvelle rédaction de l'art. 60-VI de la loi du 23 févr. 1963 reste un compromis. Le principe de la responsabilité du comptable n'est pas remis en cause ; ce sont simplement les effets qui varient selon que l'organisme dont les comptes sont jugés a ou non subi un préjudice financier. Rien ne change dans le cas où il y a préjudice : le comptable est constitué en débet. En revanche, si l'organisme n'a pas subi de préjudice, le comptable doit s'acquitter d'une somme dite « irrémissible».

Il convient de rappeler que l'introduction de la notion de préjudice financier ne figurait pas dans le projet de loi de finances rectificative qui allait aboutir à la loi n² 2011-1978 du 28 déc. 2011 (art. 90), mais a été introduite par voie d'amendement de Michel Bouvard et Charles de Courson; il est donc difficile dans ce cadre de déterminer l'exacte intention du législateur, l'amendement ayant été rapidement adopté par la commission puis en séance. Comme l'indique M. Dutheillet de Lamothe, la notion de préjudice semble toutefois reposer sur l'intuition qu'il est injuste de faire payer au comptable une somme correspondant à une contrepartie dont continue de jouir la personne publique.

La jurisprudence rendue sous l'empire des nouvelles dispositions a démontré les réelles difficultés à définir précisément, voire à critériser les hypothèses dans lesquelles le juge devait constater l'existence ou l'absence d'un préjudice financier. On doit ainsi saluer l'effort réalisé par le Conseil d'État ${ }^{11}$, sous l'impulsion de son Rapporteur public, M. Dutheillet de Lamothe, pour tenter, dans le domaine des dépenses irrégulières, d'y mettre bon ordre.

Comme l'indique le Rapporteur public, la question du fondement juridique de la dépense est ici au centre des débats. En effet, par définition, la dépense litigieuse est bien irrégulière ; à défaut en effet, aucun manquement ne pourrait être constaté et le comptable ferait l'objet d'une décision de décharge ou de quitus. II s'agit donc de déterminer les hypothèses dans lesquelles une dépense irrégulière est néanmoins juridiquement fondée.

Le Rapporteur public résume ainsi sa position : "Lorsque la dépense irrégulière a trouvé sa contrepartie, s'il y en a une, peut être regardée comme fondée juridiquement et comme ayant eu l'accord de l'ordonnateur, le juge des comptes doit en principe écarter l'existence d'un préjudice et ne pas prononcer de débet ». Cette position est ainsi corrélée à la jurisprudence de la Cour'2 qui fait reposer l'analyse du préjudice financier sur trois éléments : la réalité du service 
fait, le caractère dû ou indu de la dépense et la volonté de l'ordonnateur.

Ainsi, à bien lire les deux arrêts du Conseil d'État, éclairés par les conclusions du Rapporteur public, en se fondant sur la liste des contrôles exigés des comptables par les art. 19 et 20 RGBCP, il faudrait distinguer trois hypothèses selon la mise en œuvre d'une échelle des irrégularités commises par le comptable: en premier lieu, des vices substantiels ne seraient pas "régularisables" et causeraient un préjudice financier par nature ; en deuxième lieu, de «simples » irrégularités comptables ou "formelles » n'entraîneraient aucun préjudice financier. Enfin, dans une troisième série d'hypothèses, I'analyse des conditions dans lesquelles le comptable a rempli sa mission de contrôle de la validité de la créance doit conduire à une évaluation du fondement juridique de la dépense.

\section{Vices subtantiels révélant des cas où le manquement crée structurellement un préju- dice (sauf circonstances particu- lières)}

Selon le Conseil d'État, "Lorsque le manquement du comptable porte sur l'exactitude de la liquidation de la dépense et qu'il en est résulté un trop-payé, ou conduit à payer une dépense en l'absence de tout ordre de payer ou une dette prescrite ou non échue, ou à priver le paiement d'effet libératoire, il doit être regardé comme ayant par lui-même, sauf circonstances particulières, causé un préjudice financier à l'organisme public concerné ».

\section{a) Trop payé}

Dans ces hypothèses, considérées comme particulièrement graves, il existe une certaine identité entre le manquement et le préjudice ; autrement dit, le trop-payé révèle nécessairement un préjudice. En effet, au titre du contrôle de la validité de la dette (art. 20 RGBCP), le comptable doit s'opposer à tout paiement irrégulier ; c'est évidemment le cas en présence d'un double paiement $d^{\prime}$ une même facture ${ }^{13}$ ou d'une même somme correspondant à la même prestation ${ }^{14}$. Les mêmes solutions s'appliquent dans l'hypothèse où le comptable a payé une somme supérieure au montant effectivement dû, voire une somme qui n'était pas due ${ }^{15}$. Le trop-payé peut résulter par exemple du versement d'une rémunération ou d'une indemnité supérieure au montant prévu ${ }^{16}$, la différence correspondant en fait à une dépense personnelle ${ }^{17}$, d'un remboursement de frais supérieur au montant prévu ${ }^{18}$, d'un paiement comprenant un taux de TVA supérieur au taux normalement dû ${ }^{19}$, de la mauvaise application $d^{\prime}$ un texte fixant un mode de calcul ${ }^{20}$. La jurisprudence est identique s'agissant de paiements indus, par exemple le paiement d'impôts sans s'assurer que la collectivité publique ne bénéficie pas d'exonérations ${ }^{21}$, de cotisations sociales à I'URSSAF pour un contrat emploi solidarité alors que ces rémunérations en sont exonérées ${ }^{22}$ ou encore d'une dette communale alors qu'elle était atteinte par la prescription quadriennale $e^{23}$.

Le caractère indu d'un paiement n'est pas affecté par la réalité du service fait ou l'intention supposée de l'administration ou de l'établissement, encore moins par la disponibilité des crédits, mais repose sur le constat que les justifications, manquantes ou insuffisantes, ne confèrent pas au paiement le caractère d'une dette certaine ${ }^{24}$. Par exemple, un paiement effectué sans que la preuve ait été rapportée de l'existence d'une créance et donc que la somme était due, cause un préjudice ${ }^{25}$.

En effet, le comptable doit s'assurer de l'exactitude des calculs réalisés par l'ordonnateur ${ }^{26}$ et ce, même en l'absence de contestation du service fait $^{27}$. Il doit ainsi s'assurer que, pour effectuer les calculs, les bons tariff ${ }^{28}$ ou les bons taux ${ }^{29}$ ont été appliqués, même si les dispositions contractuelles prévoient l'application d'un autre tau ${ }^{30}$.

${ }^{12}$ CC, 26 mai 2016, DRFIP de Rhône-Alpes et du département du Rhône, n 2016-1602 ; GFP 2016, nº 6 p. 155. ${ }^{13} \mathrm{C}$. comptes, 22 mars 1989, École normale supérieure de jeunes filles de Fontenay-aux-Roses: Rec. C. comptes 36.

${ }^{14}$ C. comptes, 22 mars 1989, École normale supérieure de jeunes filles de Fontenay-aux-Roses : Rec. C. comptes 36.

${ }^{15}$ C. comptes, 25 févr. 1988, Chambre d'agriculture du Morbihan: Rec. C. comptes 46.

${ }^{16}$ C. comptes, 27 juill. 2017, Univ. de Compiègne, $n^{\circ}$ 2017-2444 (charge $n^{\circ} 1$ : GFP 2018 n² 2, p. 155.

${ }^{17}$ C. comptes, 26 févr. 1997, CNAMTS : Rev. Trésor 1998. 257.

${ }^{18}$ C. comptes, 27 juill. 2017, EPCS « Paris Sciences et Lettres-Formation », $n^{\circ}$ 2017-2332 (charge n 1) : GFP 2018 $n^{\circ} 2$, p. 151

${ }^{19}$ CE, 8 juill. 2005, Min. Éco. Fin. et Industrie c/ M. Basserie, n² 263254: GAJF, 7e éd., n 24 ; AJDA 2005. 2240 ; Rev. Trésor 2006. 147; RFDA 2006. 828

${ }^{20}$ C. comptes, 28 oct. 2016, Ch. agriculture Pas-de-Calais, $n^{\circ} 2016-3337$.

${ }^{21}$ CRC Auvergne, 5 févr. 1998, Cne de Thiers : Rev. Trésor 1998. 668.

${ }^{22}$ CRC Rhône-Alpes, 15 avr. 1998, Cne de Saint-Laurent-de-Vaux : Rev. Trésor 1998. 442.

${ }^{23}$ C. comptes, 29 sept. 2005, Cne de Vaujany: Rev. Trésor 2006. 735.

${ }^{24}$ C. comptes, 9 févr. 2017, Office public de l'habitat Dordogne Habitat, $n^{\circ} 2017-0234$ : GFP 2017 n 4, p. 142.

${ }^{25}$ C. comptes, 3 mars 2017, DRFiP Limousin, n²017-0499 (charge 13) : GFP 2017 n 6 p. 149

${ }^{26}$ C. Comptes 15 déc. 1995, SIVOM de Nouvion-en-Ponthieu, Rev. Trésor 1996. 243 ; CE 8 juill. 2005, MEFI c/ Basserie, req. $n^{\circ} 263254$.

${ }^{27}$ C. comptes, 7 avr. 1999, Cne de Saint-Denis, Rev. Trésor 1999. 775 ; RFDA 2000. 1122.

${ }^{28}$ C. comptes, 7 oct. 1993, Trésorier municipal de Marseille, Rec. C. comptes 102.

${ }^{29} \mathrm{C}$. comptes, 5 mai 1988, Cne de Garges-Lès-Gonesse, Rec. C. comptes 62.

${ }^{30} \mathrm{CE} 8$ juill. 2005, MEFI c/ Basserie, req. $n^{\circ} 263254$ 
Ainsi, un trop-versé entraîne par principe un préjudice financier ${ }^{31}$; tel est le cas par exemple d'un paiement effectué au-delà du forfait prévu par le contrat sans que les pièces justifiant ce surcoût aient été fournies pour permettre la vérification de l'exactitude de la liquidation ${ }^{32}$, du versement d'une indemnité dans un cas non prévu par le texte qui l'institue ${ }^{33}$ ou du paiement du solde d'un marché sans décompte des pénalités de retard, en l'absence de décision d'exonération ou de réduction et alors même que l'ordonnateur reconnaît sa responsabilité dans les retards ${ }^{34}$.

\section{b) Paiement non libératoire}

Les mêmes principes s'appliqueront logiquement dès lors que le paiement n'est pas libératoire ${ }^{35}$. $C^{\prime}$ est le cas par exemple lorsque le paiement est adressé à des parties autres que les créanciers ${ }^{36}$ ou leurs représentants ${ }^{37}$. Le comptable doit ainsi s'assurer qu'il n'y a pas d'opposition faite au paiement, ou des nantissements, cessions, transferts de créances $^{38}$ ou délégations de paiement ${ }^{39}$, et tenir compte des éventuelles saisies attributions dont il a été informé ${ }^{40}$. Le comptable doit encore vérifier que la dette n'a pas déjà été payée ${ }^{41}$, en totalité ${ }^{42}$ ou partiellement.

${ }^{31}$ C. comptes, 23 avr. 2014, TPG de Maine-et-Loire, n 69520.

${ }^{32}$ C. comptes, 27 sept. 2016, Univ. Versailles-Saint-Quentin-en-Yvelines, $n^{\circ} 2016-3025$ (charge $n^{\circ} 5$ ).

${ }^{33} \mathrm{C}$. comptes, 15 déc. 2016, Agence pour la mise en valeur des espaces urbains de la zone dite des cinquante pas géométriques de la Guadeloupe, n²016-3863.

${ }^{34}$ CRC île-de-France, 20 déc. 2016, Centre gérontologique "Les Abondances ", n² 2016-0038: GFP 2017 n 5, p. 151.

${ }^{35}$ C. Comptes 12 oct. 1995, Département de l'Eure, Rev. Trésor 1996. 179; 20 juill. 2017, Commune de Propriano, Rec. 143 ; GAJF $77^{e}$ éd. $n^{\circ} 28$

${ }^{36}$ C. comptes, 28 juin 2005, Cne d'Aix-en-Provence, Rev. Trésor 2006. 61.

${ }^{37}$ C. comptes 25 mai 1992, CH d'Ajaccio, Rec. C. comptes 46.

${ }^{38} \mathrm{C}$. comptes 2 oct. 1996, Cne d'Hallennes-lez-Haubourdin, Rec. C. comptes $114 ; 27$ nov. 2003, Centre national du Livre, Rev. Trésor 2004. 702.

${ }^{39} \mathrm{C}$. comptes, 10 mars 2014, Service du contrôle budgétaire et comptable placé auprès du ministre de l'écologie, $n^{\circ} 69152$

${ }^{40} \mathrm{C}$. comptes 27 nov. 2003, Centre nat. du Livre, Rec. C. comptes 100 ; Rev. Trésor 2004. 702.

${ }^{41}$ C. comptes, 6 nov. 1997, Etabl. Public national d'hosp. de Fresnes, Rev. Trésor 1998. 260 ; 28 janv. 2010, Cne d'Olette, GFP 2011. 345.

${ }^{42}$ Double paiement : C. comptes, 22 mars 1989, École normale supérieure de jeunes filles de Fontenay-auxRoses, Rec. C. comptes 36 ; 23 déc. 2013, Université Paris VI - Pierre et Marie Curie, $n^{\circ} 68234$.

${ }^{43} \mathrm{C}$. comptes, 29 mai 2018, Établissement public national d'aménagement et de restructuration des espaces commerciaux et artisanaux (Épareca), n²018-1346, GFP 2018 nº 6 p. 154.

${ }^{44}$ C. comptes, 11 déc. 2018, DDFIP d'Indre-et-Loire, n² 2018-3541; GFP 2019 n² 2 p. 152

${ }^{45}$ C. comptes, 19 juill. 2016, TPG puis DRFIP de Guadeloupe, $n^{\circ} 2016-2313$ : GFP 2017 n² 2, p. 150 ; 21 juill. 2017, Cté de cnes des Vallées du Valbonnais, n²017-2163: GFP 2018 n², p. 153 ; 21 sept. 2017, Grand por maritime de Dunkerque, $n^{\circ}$ 2017-2615: GFP $2018 n^{\circ} 2$ 2, p. 150 ; 17 mai 2018, Université de Bourgogne, $n^{\circ} 2018$ 1362, GFP 2018 nº 6 p. 156 ; 16 mai 2019, Conseil départemental de l'accès au droit (CDAD) du Val-de-Marne, $n^{\circ} 2019-1199 ;$ GFP 2019, nº 4 p. 124

${ }^{46}$ C. comptes, 20 déc. 2017, Ch. d'agriculture de la Réunion, n 2017-4077 : GFP 2018, n 4, p. 148

${ }^{47}$ C. Comptes 23 juin 1999, Caisse de crédit municipal de Nice, Rev. Trésor 2000. 365 ; C. comptes, 16 nov. 2017 Institut français de Corée du Sud, n 2017-3561: GFP 2018, n² 4, p. 150

${ }^{48}$ C. comptes, 14 févr. 2014, INRIA, n 68865 (charge no 6) : GFP 2015, n 11 p. 148 ; 18 sept. 2014, CIAS de Marsan, $n^{\circ}$ 70797: GFP 2016, n² p. 145; 11 oct. 2018, Chambre interdépartementale d'agriculture (CIA) d'îlede-France, $n^{\circ} 2018-2753$; GFP 2019 n² 2 p. 151.
Un paiement non libératoire cause en principe un préjudice à la collectivité, la somme demeurant due au véritable créancier ; toutefois, il résulte de la jurisprudence de la Cour que le juge pourra constater l'absence de préjudice dès lors que le véritable créancier ne serait pas fondé à demander un second paiement à l'établissement ${ }^{43}$. II en ira de même dès lors que la somme en cause a fait l'objet d'un remboursement ${ }^{44}$.

\section{Violation de règles qualifiées de "formelles " révélant une absence de préjudice "par nature"}

Le Conseil d'État précise en effet : "lorsque le manquement du comptable aux obligations qui lui incombent au titre du paiement d'une dépense porte seulement sur le respect de règles formelles que sont l'exacte imputation budgétaire de la dépense ou l'existence du visa du contrôleur budgétaire lorsque celle-ci devait, en l'état des textes applicables, être contrôlée par le comptable, il doit être regardé comme n'ayant pas par lui-même, sauf circonstances particulières, causé de préjudice financier à l'organisme public concerné».

Tel est le cas, par exemple et malgré une jurisprudence qui a un peu hésité, du paiement d'une subvention en l'absence de convention, l'objet de cette obligation étant d'assurer la transparence et l'information des citoyens et non le bon emploi des deniers publics ${ }^{45}$.

On relèvera pourtant que, par référence aux nomenclatures applicables, le Rapporteur public indique que "la signature d'une convention est obligatoire au-delà d'un certain montant : le préjudice ne peut être écarté que si une telle convention existe; en précisant les conditions d'emploi de la somme et les obligations du bénéficiaire, elle fonde bien la dépense ».

\section{a) Erreur d/imputation}

Si le comptable n'est pas juge de la légalité, le contrôle qu'il est tenu d'opérer sur les dépenses peut le conduire à porter une appréciation juridique sur les actes administratifs à l'origine de la créance afin de s'assurer de la correcte imputation de la dépense et de vérifier que les pièces produites sont cohérentes au regard de la catégorie de dépense ${ }^{46}$. Ainsi, les comptables doivent vérifier l'exacte imputation des dépenses, à défaut de quoi ils engagent leur responsabilité ${ }^{47}$.

Si les sommes litigieuses étaient dues, la mauvaise imputation n'a pas causé de préjudice financier pour l'établissement ${ }^{48}$. Par exemple, 
constitue un manquement sans préjudice le défaut de contrôle de l'exacte imputation des dépenses au chapitre qu'elles concernent selon leur nature ou leur objet, pour avoir à tort imputé en investissement des opérations ne répondant pas au critère de définition des immobilisations ${ }^{49} \mathrm{ou}$ l'imputation de dépenses d'investissement sur un compte de dépenses d'entretien dès lors que les dépenses en cause n'étaient pas éligibles au remboursement de TVA ${ }^{50}$.

Le Rapporteur public prend toutefois I'utile précaution de réserver le cas où l'erreur d'imputation révèle une indisponibilité des crédits ou un préjudice financier indirect ${ }^{51}$. En effet, une erreur d'imputation peut parfaitement entraîner un préjudice réel pour la personne publique en cause dès lors qu'il en résulte une dépense qui n'aurait pas dû intervenir si l'imputation avait été correcte; tel est le cas par exemple si l'erreur d'imputation aboutit à faire supporter à l'établissement des cotisations sociales indues ${ }^{52}$ ou à le priver de son droit à récupération de la TVA ${ }^{53}$. II en ira de même dès lors que la correcte imputation aurait rendu le paiement impossible ${ }^{54}$ ou si, faute de crédits suffisants sur le chapitre correspondant, la dépense n'aurait pu être réglée si le comptable avait veillé à son exacte imputation ${ }^{55}$.

\section{b) Défaut de visa du contrôleur bud- gétaire ou financier}

C'est le cas ensuite de l'absence de visa du contrôleur budgétaire (D. 2018-803 du 24 sept. 2018) : selon le Rapporteur public, il s'agit de vérifier la soutenabilité de l'opération et non sa régularité ou sa légalité.

La Cour des comptes a déjà considéré, à plusieurs reprises, que l'absence de visa du contrôleur financier n'avait pas entraîné de préjudice financier ${ }^{56}$, en particulier lorsque la réalité de l'intention de la personne publique était bien attestée et que les prestations ayant bien été réalisées ${ }^{57}$.

\section{c) Quid des dépenses "obligatoires" ?}

Même si la question n'est pas abordée par le Conseil d'État, la Cour des comptes considère que certaines opérations ne peuvent, par nature, avoir causé de préjudice financier ; c'est le cas tout particulièrement des dépenses que l'on peut qualifier d'obligatoires, elles-mêmes accompagnées des pièces justificatives requises ${ }^{58}$. L'inéluctabilité de la dépense permet ainsi de surmonter les irrégularités "formelles" qui ont accompagné son paiement.

Par exemple, n'entraîne pas de préjudice financier le paiement d'honoraires d'avocat pour la défense du maire en l'absence de délibération du conseil municipal dès lors que la commune était tenue d'accorder sa protection au maire quant il fait l'objet de poursuites pénales pour des faits n'étant pas des fautes détachables ${ }^{59}$. La même solution sera retenue, dès lors que lorsque le paiement de la prime litigieuse était inéluctable, par exemple s'agissant du versement d'une nouvelle bonification indiciaire (NBI) sans disposer de la décision individuelle de l'ordonnateur, l'agent étant de droit bénéficiaire de la NBI et son arrêté de nomination suffisant pour vérifier l'exacte liquidation de l'indemnitée ${ }^{60}$.

Le caractère obligatoire de la dépense permet même de pallier l'incompétence de l'autorité ayant ordonné la dépense ${ }^{61}$.

\section{Restent les autres hypothèses indéterminées}

"Le manquement du comptable aux autres obligations lui incombant, telles que le contrôle de la qualité de l'ordonnateur ou de son délégué, de la disponibilité des crédits, de la production des pièces justificatives requises ou de la certification du service fait, doit être regardé comme n'ayant, en principe, pas causé un préjudice financier à l'organisme public concerné lorsqu'il ressort des pièces du dossier, y compris d'éléments postérieurs aux manquements en cause, que la dépense repose sur les fondements juridiques dont il appartenait au comptable de vérifier l'existence au regard de la nomenclature, que l'ordonnateur a voulu l'exposer et, le cas échéant, que le service a été fait".

\section{a) Qualité et volonté de l'ordonnateur ou de son délégué}

Un préjudice financier doit-il être constaté lorsque la dépense, irrégulière, émane d'un ordonnateur incompétent ou a été prise au mépris des règles de compétence applicables à la personne publique en cause?

Sur le premier point et, par principe, la Cour estime que, dès lors que l'engagement de dépense est signé par une autorité qui n'est pas l'ordonnateur ou qui n'a pas reçu délégation de celui-ci, le paiement est indu et crée un préjudice ${ }^{62}$.

Dans ses conclusions, M. Dutheillet de Lamothe accorde toutefois une très grande importance à la prise en considération de la volonté de l'ordonnateur ; si celle-ci peut être clairement déduite des éléments du dossier, l'irrégularité commise par le comptable n'entraînera pas la constatation d'un préjudice financier. La Cour avait déjà jugé que le fait pour un comptable public de
${ }^{49} \mathrm{C}$. comptes, 22 juin 2017 Agence nationale des titres sécurisés (ANTS), $\mathrm{n}^{\circ} 2017$ 1685 : GFP $2017 n^{\circ} 6$ p. 150

${ }^{50}$ CRC Centre-Val de Loire, 28 mars 2017, CH George Sand, $n^{\circ}$ 2017-0001 : GFP $2018, n^{\circ} 1$ p. 154

${ }^{51}$ L'exemple classique étant l'imputation d'une dépense d'investissement d'une collectivité territoriale à la section de fonctionnement, entraînant un préjudice au regard du FCTVA.

${ }^{52}$ C. comptes, 14 févr. 2014, INRIA, n 68865 : GFP 2015 $n^{\circ} 11$ p. 141

${ }^{53}$ C. comptes, 28 oct. 2016 , Agrocampus ouest, $\mathrm{n}^{\circ} 2016$ 3318: GFP $2017 n^{\circ} 2$, p. $152 ; 10$ avr. 2015 , CNOUS, $n^{\circ} 71646$.

${ }^{54}$ C. comptes, 16 nov. 2017 Institut français de Corée du Sud, n 2017-3561: GFP 2018 $n^{\circ} 4$, p. 150.

${ }^{55}$ C. comptes, $1^{\text {er }}$ déc. 2016, Institut français d'Israël, n² 2016-3736 : GFP 2017 n4, p. 140.

${ }^{56}$ C. comptes, 18 avr. 2016, École nat. supérieure Louis Lumière, $n^{\circ} 2016-1234$ (charges nos 7 et $8 ; 27$ sept. 2016, CROUS de Rennes, $n^{\circ} 2016-3023$ (charge $n^{\circ} 6$ ); 28 avr. 2017, France Agrimer, $n^{\circ}$ 2017-1390 : GFP $2017 n^{\circ} 6$ p. 150.

${ }^{57}$ C. comptes, 19 mars 2019, Agence des systèmes $d^{\prime}$ information partagés de santé : n² 2019-0468; GFP $2019, n^{\circ} 4$ p. 123 ; 18 juill. 2019, Agence nationale de traitement automatisé des infractions (Antai) $n^{\circ} 2019$ 1777 ; GFP 2019 n 6 p. 170

${ }^{58}$ C. comptes, 25 janv. 2018, Synd. mixte dptal de traitement des déchets ménagers de l'Aisne 'Valor'Aisne', n' 2018-0042: GFP $2018 n^{\circ} 4$, p. 152.

${ }^{59}$ CRC Normandie, 7 août 2018, Commune d'Yvetot : $n^{\circ} 2018$ 15 ; GFP 2019 n 1 p. 149

${ }^{60}$ CRC Nord-Pas-de-Calais Picardie, 17 mars 2016 EHPAD de Liancourt, n'2016-0009: GFP 2017 n 1 p. 159. C. comptes, 14 déc. 2017, CH de Saint-Geoire-enValdaine, $n^{\circ}$ 2017-3799: GFP $2018 n^{\circ} 4$, p. 152

${ }^{61}$ C. comptes, 18 juill. 2019 Syndicat mixte du centre aquatique La Piscine de Fécamp (Seine-Maritime), $n^{\circ}$ 2019-1763; GFP 2019 n 6 p. 169.

${ }^{62} \mathrm{C}$. comptes, 17 mars 2016 CBCM Intérieur, $n^{\circ}$ 2016-0509 (charge $n^{\circ} 6$ ). 
prendre en compte un bon de commande signé par un agent de l'établissement en dépassement de la délégation donnée par l'ordonnateur ne signifie pas qu'il en est résulté un préjudice financier pour l'établissement ; l'établissement avait bien réceptionné le nouveau service et bénéficié d'une prestation conforme à sa demande et ses besoins ; I'ordonnateur compétent avait confirmé lors de l'instruction son consentement à la délégation de signature et à l'ordonnancement desdites dépenses ${ }^{63}$

Le juge est ainsi appelé à prendre en considération la volonté même de la personne publique ou de l'ordonnateur. Ce critère touchant à la volonté est toutefois d'un maniement extrêmement délicat et le rapporteur public a raison de considérer qu'il « est apprécié assez souplement en matière de contrat, plus rigoureusement s'agissant d'un fondement réglementaire».

Ainsi, en matière de contrats et marchés, si les prestations ont été effectivement fournies à l'organisme public en cause par le titulaire du marché et si les parties ont manifestement entendu qu'elles le soient dans les conditions contractuelles prévues par ledit marché, le juge conclura à l'absence de préjudice. La commune intention des parties peut à cet égard résulter, notamment, de la conclusion ultérieure d'un avenant de régularisation, d'un nouveau contrat ou d'une convention de transaction conclus avec le titulaire du marché ${ }^{64}$. La commune intention des parties de contracter pour la réalisation des prestations portées sur la facture est suffisamment établie par le bon de commande produit lors de la phase non contentieuse de l'examen des comptes; de ce fait, le manquement du comptable en matière de dépenses n'a pas causé de préjudice financier à l'État ${ }^{65}$.

En revanche, en matière de primes et indemnités, il ne résulte pas de la jurisprudence qu'une manifestation de volonté de l'auteur de la dépense suffise, par elle-même, à considérer que cette dernière est due. Encore faut-il que celle-ci ait un fondement juridique probant permettant un paiement régulier. Ni la certification du service fait ni la signature des bordereaux de mandat par l'ordonnateur ne sont de nature à démontrer l'absence de préjudice, quand bien même le directeur ayant le pouvoir de prendre la décision attributive et l'ordonnateur constituent une seule et même personne ${ }^{66}$.

Par suite, une délibération prise a posteriori, visant à régulariser des primes accordées dans des conditions irrégulières, n'est pas susceptible d'effacer le préjudice subi par l'établissement au moment du paiement; en matière de primes et d'indemnités, l'intention, a fortiori exprimée à titre rétroactif, est donc irrégulière et ne saurait suffire à régulariser les dépenses intervenues ; la volonté de l'ordonnateur d'attribuer les indemnités ne permet pas davantage d'établir la volonté de l'assemblée délibérante ${ }^{67}$.

La même solution est retenue en cas de paiement de primes en l'absence de décisions individuelles d'attribution; si, en première instance, le juge avait conclu à l'absence de préjudice financier au motif que l'ordonnateur avait bien signé les mandats et bordereaux de mandat, les fiches de paie mentionnant le nom des bénéficiaires, la nature des primes et les éléments de leur liquidation, la Cour a estimé que ces signatures ne pouvaient pallier l'absence de l'édiction, par le même ordonnateur, des arrêtés individuels d'attribution des primes litigieuses ${ }^{68}$.

La situation est plus complexe encore s'agissant de la prise en compte des règles de compétence applicables à la personne publique en cause : par exemple, une dépense est payée par le comptable sur le fondement d'une décision de I'ordonnateur alors que le droit applicable à l'organisme public en cause exigeait l'intervention de l'assemblée délibérante.

La Cour des comptes considère que des paiements s'appuyant sur des délibérations prises par une autorité incompétente doivent être considérés comme indus et entraînent dès lors un préjudice $^{69}$. Elle estime que l'engagement d'une dépense par une autorité incompétente révèle une dépense dépourvue de fondement juridique, alors même que la prestation avait été fournie ${ }^{70}$.

Toutefois, le Rapporteur public rappelle que " le comptable n'a pas à vérifier la correcte application des règles de répartition des compétences ou le respect des délégations (CE, 4 mai 2018, Min de l'action et des comptes publics, $n^{\circ} 410880^{71}, T$. ; CE, 28 décembre 2018, SDIS de la Gironde, $n^{\circ} 410113^{72}$, T.), s'agissant de l'engagement administratif de la dépense. Autrement dit, si la nomenclature exige de lui qu'il vérifie l'existence d'une délibération du conseil d'administration autorisant, par exemple la signature d'un contrat, comme c'est généralement le cas, par exemple, pour les contrats de partenariat, le préjudice ne sera écarté que si cette délibération est produite devant le juge des comptes, ce qui établira à la fois fondement et volonté. Si en revanche un tel justificatif n'est pas exigé, le comptable n'avait pas à s'intéresser à l'accord de I'organe délibérant et aurait dû exécuter le mandat de l'ordonnateur y compris en cas d'absence illégale de cette délibération. II n'y a alors pas de 
raison de rechercher la volonté de l'organisme au stade de l'examen du préjudice ».

La référence à la nomenclature applicable est redoutable et renvoie le pouvoir réglementaire a ses responsabilités : à lui d'indiquer clairement dans la nomenclature jusqu'où le comptable doit aller dans son contrôle; et le silence de la nomenclature sur ce point pourra être interprété comme une sorte de tolérance au bénéfice du comptable qui ne fera pas l'objet d'une mise en débet. En revanche, l'illégalité de la décision subsiste, dissimulant mal soit une insuffisance du contrôle de légalité s'agissant des collectivités territoriales et des établissements publics, soit une volonté clairement assumée de se soustraire aux règles de répartition interne des compétences au sein des organismes publics.

\section{b) Disponibilité des crédits}

On sait que les crédits budgétaires constituent les limites des dépenses consenties et les dépenses faites en dépassement de ces crédits sont nécessairement irrégulières ${ }^{73}$. II en résulte que les dépenses publiques ne peuvent être payées que dans la limite des crédits disponibles, avec pour seule exception le cas des crédits évaluatifs, et seulement pour l'État. Toute dépense payée en méconnaissance de ces règles engage la responsabilité du comptable $e^{74}$. Le contrôle de la disponibilité des crédits incombe au comptable en application de l'art. 19 RGBCP, sans se confondre avec le contrôle de la validité de la dépense, qui relève de l'art. $20 \mathrm{RGBCP} 75$.

M. Dutheillet de Lamothe avoue avoir hésité à proposer d'ajouter l'indisponibilité des crédits à la liste des cas où le manquement crée structurellement un préjudice. II l'exclut toutefois au motif que "l'article 7 de loi organique sur les lois de finances $n^{\circ}$ 2001-692 du 1er août 2001 a consacré une importante fongibilité des crédits entre les titres ». On peut donc en déduire qu'en principe le paiement sur crédits indisponibles entraîne un préjudice financier, sauf mise en œuvre de la fongibilité asymétrique des crédits telle que prévue par la LOLF.

\section{c) Production des pièces justificatives}

A rebours de ce qui était indiqué s'agissant du renvoi à la nomenclature pour apprécier la répartition interne des compétences des organismes publics, les manquements résultant du défaut ou de l'insuffisances des pièces justificatives ne sont pas considérés comme causant, par principe, un préjudice financier ${ }^{76}$. Cette position, pour raisonnable qu'elle soit, démontre les limites de la référence aux nomenclatures puisque ce sont pré- cisément elles qui énumèrent les pièces que le comptable doit exiger avant tout paiement.

Quoi qu'il en soit, le juge des comptes a su faire preuve d'interprétations constructives pour pallier le défaut de pièces justificatives, notamment en présence de simples erreurs formelles : par exemple, absence de date sur des mandats par ailleurs pleinement justifiés ${ }^{77}$, incohérences entre l'ordre de mission et le mandat de paiement quant aux dates et heures de déplacements ${ }^{78}$. Enfin, la pièce faisant défaut doit présenter un caractère substantiel ; à défaut, le juge constatera l'absence de préjudice financier, par exemple lorsque la pièce manquante est un simple avis consultatif?

Le juge conclut également à l'absence de préjudice dès lors que le comptable avait une connaissance précise de la nature et de l'objet de l'opération ${ }^{80}$, que les justificatifs produits attestent de la réalité des prestations ${ }^{81}$ ou lorsqu'aucun élément ne permet de mettre en doute l'exécution du service ni d'établir que les frais ainsi payés aient été indus ${ }^{82}$

\section{d) Certification du service fait}

Le constat de l'existence matérielle du service

fait, c'est-à-dire de l'existence même de la prestation, constitue une condition élémentaire de la constatation de l'absence de préjudice financier. Autrement dit, si la dépense réalisée n'a entraîné aucune contrepartie, l'irrégularité a nécessairement entraîné un préjudice financier. Les deux autres critères (dépense due ou non et volonté de l'ordonnateur) sont ici présentés comme inopérants, sans toutefois que l'on comprenne vraiment pourquoi.

En effet, au-delà des dépenses sans contrepartie directe (aides publiques, subventions, primes ${ }^{83}$ ), pourquoi la volonté de l'ordonnateur, clairement exprimée sous sa seule responsabilité (illusoire, mais on y reviendra), ne pourrait-elle exonérer le comptable d'avoir à rembourser une somme n'ayant entraîné aucune contrepartie ? N'est-il pas injuste de mettre en débet un comptable pour avoir versé des rémunérations complémentaires fictives (donc sans contrepartie pour la personne publique en cause) quand l'ordonnateur affirmait le contraire ${ }^{84}$ ? II existe certes un manquement et un préjudice ; mais ne peut-on considérer que la volonté de l'ordonnateur a brisé le lien de causalité entre le manquement du comptable et le préjudice subi ? On semble revenir ici à une conception purement matérielle du préjudice qui avait pourtant été exclue par le Rapporteur public qui considérait qu'il « ne faut pas se demander, dans l'absolu, si la collectivité

\footnotetext{
${ }^{73} \mathrm{C}$. comptes, BAS de Matour, GAJF 7e éd. $n^{\circ} 27$.

${ }^{74}$ C. comptes, 28 janv. 2010, Lycée polyvalent de l'image et du son d'Angoulême, n 56774 GFP 2011. 167

${ }^{75}$ C. comptes, 24 oct. 2013 Maison de retraite «SaintFrançois » à Vermenton, $n^{\circ} 67984$.

${ }^{76}$ La Cour des comptes retient toutefois la solution contraire C. comptes, 21 juill. 2016 , Cne de Risoul, $n^{\circ} 2016-2429$ : AJDA 2017. 928 ; Gestion et fin. publ. $2017 n^{\circ}$ 2, p. 154.

${ }^{77}$ C. comptes, 16 oct. 2015 , Autorité de régulation des activités ferroviaires, $n^{\circ} 72739$ : GFP 2016 n² 2 p. 139.

${ }^{78}$ C. comptes, 27 sept. 2016 CROUS de Rennes, $n^{\circ} 2016$ 3023 (charge $n^{\circ} 3$ ).

${ }^{79}$ C. comptes, 27 oct. 2017, INPI, n² 2017-3317 (charges nos 1 et 2) : GFP $2018 n^{\circ} 2$ p. 153.

${ }^{80}$ C. comptes, 24 oct. 2013 , ENSP, n 67942: GFP 2015, n 11 p. 145.

${ }^{81}$ C. comptes, 26 mai 2016 DRFiP Rhône-Alpes, n² 2016 1602 (charge $n^{\circ} 20$ ).

${ }^{82}$ C. comptes, 17 nov. 2016, Cté de Cnes des Hauts du Lyonnais, $n^{\circ}$ 2016-3283 : GFP 2017 n $^{\circ} 4$ p. 143.

${ }^{83}$ Dans ces cas, en effet, aucune contrepartie directe ne peut être identifiée ; toutefois, par exemple, le bénéficiaire d'une subvention doit utiliser celle-ci conformément à son objet ; le bénéficiaire d'une prime doit justifier de son activité professionnelle...

${ }^{84}$ Situation illustrée par C. comptes, 15 avril 2019 Université Lille 3 (Nord), $n^{\circ}$ 2019-1033; GFP 2019, n 4 p. 124.
} 
publique souffre financièrement : la recherche du préjudice doit partir d'une interrogation sur les conséquences qu'a eu le manquement du comptable par lui-même ».

Quoi qu'il en soit, il existe sur ce point une cohérence parfaite entre la jurisprudence de la Cour des comptes et de son juge de cassation : I'absence de contrepartie est nécessairement source d'un préjudice financier pour l'organisme public.

A l'inverse, l'existence d'un service fait ne suffit pas à faire considérer que le paiement irrégulier de la dépense n'a pas causé de préjudice ${ }^{85}$. Si les dépenses en cause correspondent bien à des prestations effectivement réalisées au profit de l'établissement, cette circonstance ne suffit pas à elle seule à justifier l'absence de préjudice financier, dès lors en particulier qu'il est impossible d'établir avec certitude le montant auquel aurait pu s'élever un hypothétique paiement régulier de telles prestations par l'établissement ${ }^{86}$.

Mais le juge peut toutefois considérer que la dépense en cause étant la contrepartie d'un service fait ou de prestations effectivement réalisées et le paiement de la facture étant bien dû, il y a lieu de constater l'absence de préjudice ${ }^{87}$. C'est tout particulièrement le cas en matière de rémunérations; ainsi, l'absence de référence à la délibération relative à l'emploi à pourvoir dans le contrat de l'agent si elle rend son paiement irrégulier ne crée par un préjudice à la collectivité dès lors que le contrat signé a donné lieu à la réalisation effec-

\footnotetext{
${ }^{85}$ C. comptes, 24 oct. 2013, ENSP, n 67942 : GFP 2015, n 11 p. 145 (sol. impl.) ; 22 janv. 2015, Cne Saint-Josse $n^{\circ} 71644$; 18 avr. 2016, ENS Louis Lumière, $n^{\circ}$ 2016-1234 (charge nº 6) ; 17 nov. 2016, SITTOMAT, $n^{\circ}$ 2016-3279.

${ }^{86} \mathrm{C}$. comptes, 19 déc. 2013, Service hydrographique et océanographique de la marine, $n^{\circ} 68201$.

${ }^{87}$ C. comptes, 8 juill. 2013, Ét. public d'aménagement de la défense (EPAD), nº 67413: GFP 2014. 166 ; 28 avr. 2014, TPG de Haute-Corse, nº 69617 ; 25 nov. 2013, Centre d'expérimentation pédagogique de Florac, $n^{\circ} 67620$.

${ }^{88}$ C. comptes, 19 déc. 2016, GIP « Formation continue et insertion professionnelle » de Paris, $n^{\circ} 2016-3851$ : GFP $2017 n^{\circ} 4$ p. 145

${ }^{89}$ C. comptes, 13 févr. 2013, TPG du Cantal, n 65085, GFP 2014, n 7/8 p. 166.

${ }^{90} \mathrm{C}$. comptes, 7 avr. 2014, Office nat. interprof. plantes à parfum, aromatiques et médicinales $n^{\circ} 69245$

${ }^{91}$ C. comptes, 26 mai 2016, DRFiP Rhône-Alpes, $n^{\circ}$ 2016-1602 (charge $n^{\circ}$ 9). C. comptes, 3 mars 2017, DRFiP Limousin, $n^{\circ} 2017-0499$ (charge nos 8 et 11) : GFP 2017, nº 6, p. 149.

92 C. comptes, 4 mai 2016, Ét. publ. d'aménagement Orly, Rungis, Seine amont (EPA ORSA), n²016-0732 (charge $n^{\circ} 1$ ).

${ }^{93}$ C. comptes, 24 janv. 2012, TPG de l'Aude, nº 62766, GFP déc. 2013, p. 78.

${ }^{94} \mathrm{C}$. comptes, 26 oct. 2017, Centre pour les études en France de Bogota, n 2017-3210 : 13 déc. 2017, Ch. dptale d'agriculture du Puy-de-Dôme, n²017-4020 : GFP 2018 n 4, p. 155

${ }^{95}$ C. comptes, 23 avr. 2007, GIP ARH de Picardie, Rev. Trésor 2008. 772 ; 12 juill 2013, Université de Corse, $n^{\circ} 67387$, GFP 2014, $n^{\circ} 7 / 8$ p. 164

${ }^{96}$ CE, 21 mars 2001, M. Morel, req. $n^{\circ} 195508$; C. comptes, 26 févr. 2009, CAT de La Bréotière, GFP 2010. 100.

${ }^{97}$ C. comptes 9 mars 1995, Chancellerie de l'académie de Corse, Rec. C. comptes 6.

${ }^{98}$ C. comptes, 6 juill. 2000, Port autonome de Dunkerque, Rev. Trésor 2001. 184.

${ }^{99} \mathrm{C}$. comptes, 6 déc. 2004. Université de Paris-Sud, Rev. Trésor 2005, 611 et nos obs.

${ }^{100} \mathrm{C}$. comptes, 27 mars 2014, Institut nat. des jeunes aveugles, $n^{\circ} 69009$.

${ }^{101}$ C. comptes, 17 mars 2016, CBCM Intérieur, n²016-0509 (charge n³).
}

tive d'un travail par la personne engagée et que l'ordonnateur confirme sa volonté de contracter pour recruter cette personne ${ }^{88}$.

S'agissant de la certification du service fait, la jurisprudence financière fait preuve d'un assez grand formalisme ; ainsi, il convient que l'ordonnateur accompagne les pièces non dématérialisées de la mention explicite " service fait " ${ }^{89}$, éventuellement, sur le bordereau d'émission des ordres de dépenses ${ }^{90}$, sur les mandats à proprement parler ou sur les factures ${ }^{91}$. Les mentions " ordre de payer » et «bon pour paiement » ne sauraient être substituées à la mention « pour valoir certification du service fait et ordre de payer » en ce qu'elles ne certifient pas le service fait ${ }^{92}$. En l'absence de pièces jointes portant la mention "service fait " ou de certification de la part de l'ordonnateur, le comptable doit suspendre le paiement ${ }^{93}$.

La Cour en déduit qu'un paiement, en l'absence d'attestation de service fait signée par l'ordonnateur, génère un préjudice dès lors que le paiement est indu, les créances n'étant pas certaines ${ }^{94}$.

\section{e) Le caractère public de la dépense}

Bien que n'étant abordée ni par le Conseil d'État ni par son rapporteur public, la question se pose de savoir si le paiement d'une dépense n'ayant pas un caractère public entraîne ou non un préjudice financier pour l'organisme public en cause.

Alors même que rien dans le RGBCP ne l'indique expressément, le juge des comptes considère que les comptables doivent s'assurer du caractère public de la dépense ${ }^{95}$, lequel ne se présume pas $^{96}$. En effet, les comptables n'ont pas à payer des dépenses à caractère personnel comme le costume de cérémonie d'un recteur ou la taxe d'enlèvement des ordures ménagères grevant un logement de fonction ${ }^{97}$, I'adhésion d'un responsable d'un établissement public au Rotary ${ }^{98}$ ou d'un professeur à une société savante ${ }^{99}$.

Le juge des comptes a affirmé clairement que des dépenses qui ne peuvent être régulièrement financées par une personne publique lui portent un préjudice financier lorsqu'elles sont payées ${ }^{100}$. C'est le cas par exemple de dépenses effectuées dans des restaurants, bars et établissements de nuit dont il n'est pas établi qu'elles étaient liées à des frais de réception ou de représentation du ministre ; en effet, les attestations émanant du cabinet du ministre sont trés générales : le juge a déduit de l'insuffisance des pièces justificatives l'existence d'un manquement entraînant un préjudice financier ${ }^{101}$. 
En revanche, le Conseil d'État estime qu'il n'appartient pas au comptable de contrôler si les dépenses d'une collectivité territoriale peuvent être rattachées aux compétences de ladite collectivité ${ }^{102}$. II en va de même pour les établissements publics ${ }^{103}$.

La question demeure donc entière de savoir s'il s'agit, en cas de manquement, d'une simple irrégularité formelle ou d'un vice substantiel.

\section{Conclusion}

De ce qui précède résulte un sentiment ambivalent qui nécessite très certainement de réfléchir, une fois encore, à l'évolution des textes applicables s'agissant de la responsabilité du comptable et, plus largement, du gestionnaire public.

Tout d'abord, l'introduction de la notion de préjudice financier en 2011, pour salutaire qu'elle ait été, a entraîné un surcroît de complexité, laquelle ne fait jamais bon ménage avec l'État de droit : le manquement commis par le comptable sera ainsi plus ou moins «puni » en fonction de sa gravité (vices substantiels ou irrégularités formelles); et l'on reste arc-bouté sur la distinction entre un comptable seulement chargé d'assurer la "régularité" des opérations sans jamais se prononcer sur leur "légalité". Outre le fait que cette distinction est souvent extrêmement difficile à réaliser, elle aboutit à des situations parfaitement ubuesques: comme l'indique M. Dutheillet de Lamothe dans ses conclusions, " les contrôles du comptable peuvent le conduire à s'interroger sur certaines questions de légalité (...) mais au-delà des contrôles comptables, la dépense décidée par l'ordonnateur doit être payée, même si elle n'est pas due, même si elle est illégale. L'ordonnateur engage sa responsabilité propre, et le comptable n'a pas pour mission de se faire le censeur de ses choix ».

Cette sur-valorisation du rôle de l'ordonnateur pourrait se concevoir dans un système où l'absence de responsabilité de l'un (le comptable) était compensée par la responsabilité de l'autre (l'ordonnateur). Or, chacun sait que tel n'est pas le cas, le système retenu aboutissant ainsi à l'application "régulière" de décisions illégales, sans que nul n'en assume la responsabilité.

Au final, en l'état actuel du droit, la volonté de I'ordonnateur, magnifiée par le rapporteur public, ne peut être l'alpha et l'omega de notre système de gestion publique.
${ }^{102}$ CE, 30 juill. 2003, Marty,
$n^{\circ} 232430$ : Lebon T. $723 ;$
Rec. C. comptes $174 ;$ Rev.
Trésor $2004.135 ;$ RFDA
2004. 823.
${ }^{103}$ C. comptes, 10 mai 2006, EP
chargé de l'aménagement
des rives de l'Étang de

Berre : Rev. Trésor 2007. 163. 